ARTICLE

\title{
Structural insights into multiplexed pharmacological actions of tirzepatide and peptide 20 at the GIP, GLP-1 or glucagon receptors
}

\author{
Fenghui Zhao 1,2,10, Qingtong Zhou ${ }^{3,10}$, Zhaotong Cong3,10, Kaini Hang4,10, Xinyu Zou ${ }^{5,10}$, Chao Zhang ${ }^{4}$, \\ Yan Chen ${ }^{3}$, Antao Dai ${ }^{6}$, Anyi Liang 5 , Qianqian Ming ${ }^{7}$, Mu Wang ${ }^{4}$, Li-Nan Chen7, Peiyu Xu (1) ${ }^{2}$, Rulve Chang ${ }^{1}$,

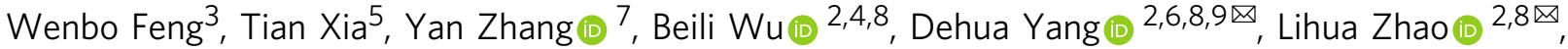 \\ H. Eric Xu (D) ${ }^{2,8 凶}$ \& Ming-Wei Wang (1) 1,2,3,4,6,8,9凶
}

Glucose homeostasis, regulated by glucose-dependent insulinotropic polypeptide (GIP), glucagon-like peptide-1 (GLP-1) and glucagon (GCG) is critical to human health. Several multi-targeting agonists at GIPR, GLP-1R or GCGR, developed to maximize metabolic benefits with reduced side-effects, are in clinical trials to treat type 2 diabetes and obesity. To elucidate the molecular mechanisms by which tirzepatide, a GIPR/GLP-1R dual agonist, and peptide 20, a GIPR/GLP-1R/GCGR triagonist, manifest their multiplexed pharmacological actions over monoagonists such as semaglutide, we determine cryo-electron microscopy structures of tirzepatide-bound GIPR and GLP-1R as well as peptide 20-bound GIPR, GLP-1R and GCGR. The structures reveal both common and unique features for the dual and triple agonism by illustrating key interactions of clinical relevance at the near-atomic level. Retention of glucagon function is required to achieve such an advantage over GLP-1 monotherapy. Our findings provide valuable insights into the structural basis of functional versatility of tirzepatide and peptide 20.

\footnotetext{
${ }^{1}$ School of Pharmacy, Fudan University, Shanghai, China. ${ }^{2}$ The CAS Key Laboratory of Receptor Research, Shanghai Institute of Materia Medica, Chinese Academy of Sciences, Shanghai, China. ${ }^{3}$ Department of Pharmacology, School of Basic Medical Sciences, Fudan University, Shanghai, China. ${ }^{4}$ School of Life Science and Technology, ShanghaiTech University, Shanghai, China. ${ }^{5}$ School of Artificial Intelligence and Automation, Huazhong University of Science and Technology, Wuhan, China. ${ }^{6}$ The National Center for Drug Screening, Shanghai Institute of Materia Medica, Chinese Academy of Sciences, Shanghai, China. 7 Department of Biophysics and Department of Pathology of Sir Run Run Shaw Hospital, Zhejiang University School of Medicine, Hangzhou, China.

${ }^{8}$ University of Chinese Academy of Sciences, Beijing, China. ${ }^{9}$ Research Center for Deepsea Bioresources, Sanya, Hainan, China. ${ }^{10}$ These authors contributed

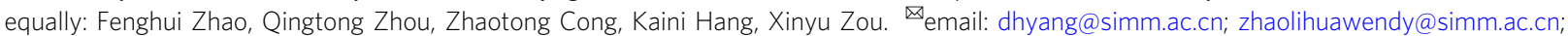
eric.xu@simm.ac.cn; mwwang@simm.ac.cn
} 
G lucose-dependent insulinotropic polypeptide (also known as gastric inhibitory peptide, GIP), glucagon-like peptide1 (GLP-1) and glucagon (GCG) are peptide hormones responsible for glucose homeostasis ${ }^{1,2}$. Their cognate receptors, GIPR, GLP-1R and GCGR, belong to class B1 G protein-coupled receptor (GPCR) family. Successful application of various GLP-1 mimetics to treat type 2 diabetes mellitus (T2DM) and obesity highlights the clinical value of this group of drug targets ${ }^{3}$. However, development of GIPR- and GCGR-based therapeutics has encountered drawbacks due to the complexity of physiology associated with GIP and GCG ${ }^{4-6}$. For example, GIP stimulates insulin secretion but also increases GCG levels ${ }^{7,8}$, while the latter has a parallel role in elevating energy expenditure and blood glucose ${ }^{9}$.

It was reported that the weight loss property of most GLP-1 analogs, except for semaglutide administered subcutaneously once weekly of smoother pharmacokinetics, is hampered by the therapeutic window because of the dose-dependent sideeffects $^{10,11}$. Chimeric peptides consisting of amino acids from GIP and GLP-1 were then designed to maximize their metabolic benefits ${ }^{12}$. Additional consideration was given to GCG for its role in energy expenditure ${ }^{13}$. Therefore, multi-targeting or unimolecular peptides possessing combinatorial agonism at GIPR, GLP-1R and GCGR have been extensively explored and more than a dozen peptides including two GIPR/GLP-1R dual agonists, ten GLP-1R/GCGR dual agonists and five GIPR/GLP-1R/GCGR triagonists have entered into clinical development (Supplementary Fig. 1a and Supplementary Table 1$)^{14}$. Of them, two pioneered multi-targeting agonists, tirzepatide (LY3298176) and peptide 20 (MAR423) have attracted significant attention from both academic and industrial communities (Fig. 1a). Tirzepatide is an investigational once-weekly GIPR/GLP-1R dual agonist ${ }^{15}$ with a profound therapeutic superiority in reducing blood glucose and body weight beyond several approved drugs such as semaglutide $^{16}$ and dulaglutide ${ }^{17}$ in multiple head-to-head clinical trials. Peptide 20, a GIPR/GLP-1R/GCGR triagonist (phase 1 clinical trials completed $)^{18-20}$ with balanced potency at the three receptors, is evolved from a GLP-1R/GCGR dual agonist ${ }^{21}$ through iterative sequence refinement and modification (Supplementary Fig. 1b) ${ }^{22}$. It reversed glucose dysregulation without detrimental effects on metabolically healthy animals and reduced body weight, lowered fasting blood glucose, decreased glycosylated hemoglobin ( $\mathrm{HbA1C}$ ), improved glucose tolerance, and protected pancreatic islet architecture in diabetic fatty Zucker rats $^{22-24}$.

The aim of this work is to understand molecular mechanisms of the dual and triple agonism conferred by tirzepatide and peptide 20. Thus, we determine five cryo-electron microscopy (cryo-EM) structures, including GIPR and GLP-1R bound with tirzepatide and GIPR, GLP-1R and GCGR bound with peptide 20, all in complex with $G_{s}$ proteins at global resolutions of $3.4 \AA$, $3.4 \AA, 3.1 \AA$, $3.0 \AA$ and $3.5 \AA$, respectively. Integrated with pharmacological and clinical data, this work reveals the structural basis of peptide recognition by each receptor and provides important information for the design of better drugs through combinatorial agonism.

\section{Results}

Overall structure. The tirzepatide-GIPR- $\mathrm{G}_{\mathrm{s}}$, tirzepatide-GLP$1 R-G_{s}$, peptide $20-G I P R-G_{s}$, peptide $20-G L P-1 R-G_{s}$ and peptide $20-G C G R-G_{s}$ structures were determined by the single-particle cryo-EM approach with overall resolutions of $3.4 \AA$, $3.4 \AA$, $3.1 \AA$, $3.0 \AA$, and $3.5 \AA$, respectively (Fig. 1b, c, Supplementary Figs. $2-7$, Supplementary Tables 2, 3). Apart from the $\alpha$-helical domain of $\mathrm{Ga}_{s}$, the presence of bound tirzepatide and peptide 20 , individual receptor and heterotrimeric $G_{s}$ in respective complex was clearly visible in all five EM maps, thereby allowing unambiguous modeling of the secondary structure and side chain orientation of all major components of the complexes (Supplementary Fig. 6).

Tirzepatide has two non-coded amino acid residues at positions 2 and 13 (Aib, $\alpha$-aminoisobutyric acid), and is acylated on $\mathrm{K} 20^{\mathrm{P}}$ (P indicates that the residue belongs to the peptide) with a $\gamma$ Glu-2×OEG linker and C18 fatty diacid moiety. The first 30 and 29 amino acids of tirzepatide were modeled for the tirzepatide- $G I P R-G_{s}$ and tirzepatide-GLP- $1 R-G_{s}$ complexes, respectively.

Peptide 20 contains two modifications: $\mathrm{A}_{2}{ }^{\mathrm{P}}$ with $\mathrm{Aib}$ and $\mathrm{K} 10^{\mathrm{P}}$ that is covalently attached by a 16-carbon acyl chain (palmitoyl; 16:0) via a gamma carboxylate $(\gamma \mathrm{E} \text { spacer })^{22}$. The $\gamma \mathrm{E}$ spacer and palmitic acid (C16:0) were well resolved in the final models of peptide $20-G L P-1 R-G_{s}$ and peptide $20-G C G R-G_{s}$, while only the $\gamma \mathrm{E}$ spacer was modeled for peptide $20-\mathrm{GIPR}-\mathrm{G}_{\mathrm{s}}$ with highresolution features. The first 30, 29, and 28 amino acids of peptide 20 were modeled for the peptide $20-G I P R-G_{s}$, peptide $20-G L P-$ $1 R-G_{s}$ and peptide $20-G C G R-G_{s}$ complexes, respectively.

As shown in Fig. 2a, the tirzepatide-GIPR- $G_{s}$ and peptide 20-GIPR- $G_{s}$ complex structures closely resembled that of the GIP-GIPR-G $\mathrm{G}_{\mathrm{s}}$ comple ${ }^{25}$ with Ca root mean square deviation (RMSD) values of 0.5 and $0.4 \AA$, respectively. Notable conformational differences were observed in the positions of peptide C-terminal half and the surrounding extracellular loop 1 (ECL1) and extracellular domain (ECD), indicative of GIPR-associated ligand specificity. Through two mutations $\left(\mathrm{M}_{1} 4^{\mathrm{P}} \mathrm{L}\right.$ and $\left.\mathrm{H} 18^{\mathrm{P}} \mathrm{A}\right)$, the dense contacts between ECL1 (residues 194 to 211) and GIP were disrupted by peptide 20 , as seen from the buried surface area that decreased from $406 \AA^{2}$ for GIP to $278 \AA^{2}$ for peptide 20 . Consequently, ECL1 adopted a more relaxed conformation, making peptide 20 straighter by shifting its tip toward the transmembrane domain (TMD) core by $4.2 \AA$ (measured by the $\mathrm{Ca}$ of $\mathrm{L}^{2} \mathrm{7}^{\mathrm{P}}$ ). Similar movement was also seen for the C-terminal half of tirzepatide $\left(2.1 \AA\right.$ measured by the $\mathrm{Ca}$ of $\left.\mathrm{I} 27^{\mathrm{P}}\right)$. As far as the $\mathrm{N}$ terminus is concerned, GIP and tirzepatide were stabilized by multiple strong contacts with the TMD core through a common $\mathrm{N}$ terminus $\left(\mathrm{Y}^{\mathrm{P}}-\mathrm{A} / \mathrm{Aib}^{\mathrm{P}}-\mathrm{E} 3^{\mathrm{P}}\right)$, while that of peptide $20\left(\mathrm{H}^{\mathrm{P}}-\mathrm{Aib}_{2} \mathrm{P}_{-} \mathrm{Q}^{\mathrm{P}}\right)$ formed weaker interactions with the TMD core by abolishing the hydrogen bond with Q224 $4^{3.37 b}$ (class B GPCR numbering in superscript ${ }^{26}$, salt bridge with $\mathrm{R} 183^{2.60 \mathrm{~b}}$ and hydrophobic contacts with V227.40b (Fig. 2b). Such deficiency of peptide 20 was rescued by the introduction of $\mathrm{T}^{\mathrm{P}}$ (hydrogen bond with $\mathrm{R} 190^{2.67 \mathrm{~b}}$ ), lipidated $\mathrm{K} 10^{\mathrm{P}}$ and $\mathrm{Y} 13^{\mathrm{P}}$ that contributed additional contacts with GIPR not observed in GIP25. The hydrogen bond between $\mathrm{T}^{\mathrm{P}}$ and $\mathrm{R} 190^{2.67 \mathrm{~b}}$ was also found in the tirzepatide-GIPR- $\mathrm{G}_{\mathrm{s}}$ complex.

The structures of tirzepatide- and peptide 20-bound GLP-1R are highly similar to that bound by GLP-127, with Ca RMSD of $0.8 \AA$ and $0.7 \AA$, respectively (Fig. 2c). The bound peptides (GLP1 , tirzepatide and peptide 20) overlapped well and penetrated into the receptor TMD core by an identical angle and orientation, thereby exploiting a similar ligand recognition pattern for most residues except for a few positions that have distinct amino acids (Fig. 2c, Supplementary Tables 4,5$)$. The substitution $\left(\mathrm{Y}_{10} \mathrm{P}\right.$ in tirzepatide) and modification (lipidated $\mathrm{K}_{10} \mathrm{P}$ in peptide 20) stabilized the binding of dual and triple agonists by newly-formed interactions with residues surrounding the TM1-TM2 cleft, a phenomenon unseen in the case of GLP-127. Meanwhile, some favorable interactions in GLP-1 recognition were absent for both tirzepatide $\left(\mathrm{Y} 13^{\mathrm{P}} \mathrm{A}\right.$ decreased the hydrophobic interactions with TM1, E21 ${ }^{\mathrm{P}} \mathrm{A}$ broke the hydrogen bond with Q210 ${ }^{\mathrm{ECL}}$ ) and peptide $20\left(\mathrm{E}^{\mathrm{P}} \mathrm{Q}\right.$ eliminated the salt bridge with $\left.\mathrm{R} 190^{2.60 \mathrm{~b}}\right)$ (Fig. 2d). Interestingly, the residues at multiple positions $(12,16$, $17,20,21,24$ and 28) of the multi-targeting agonists are highly 

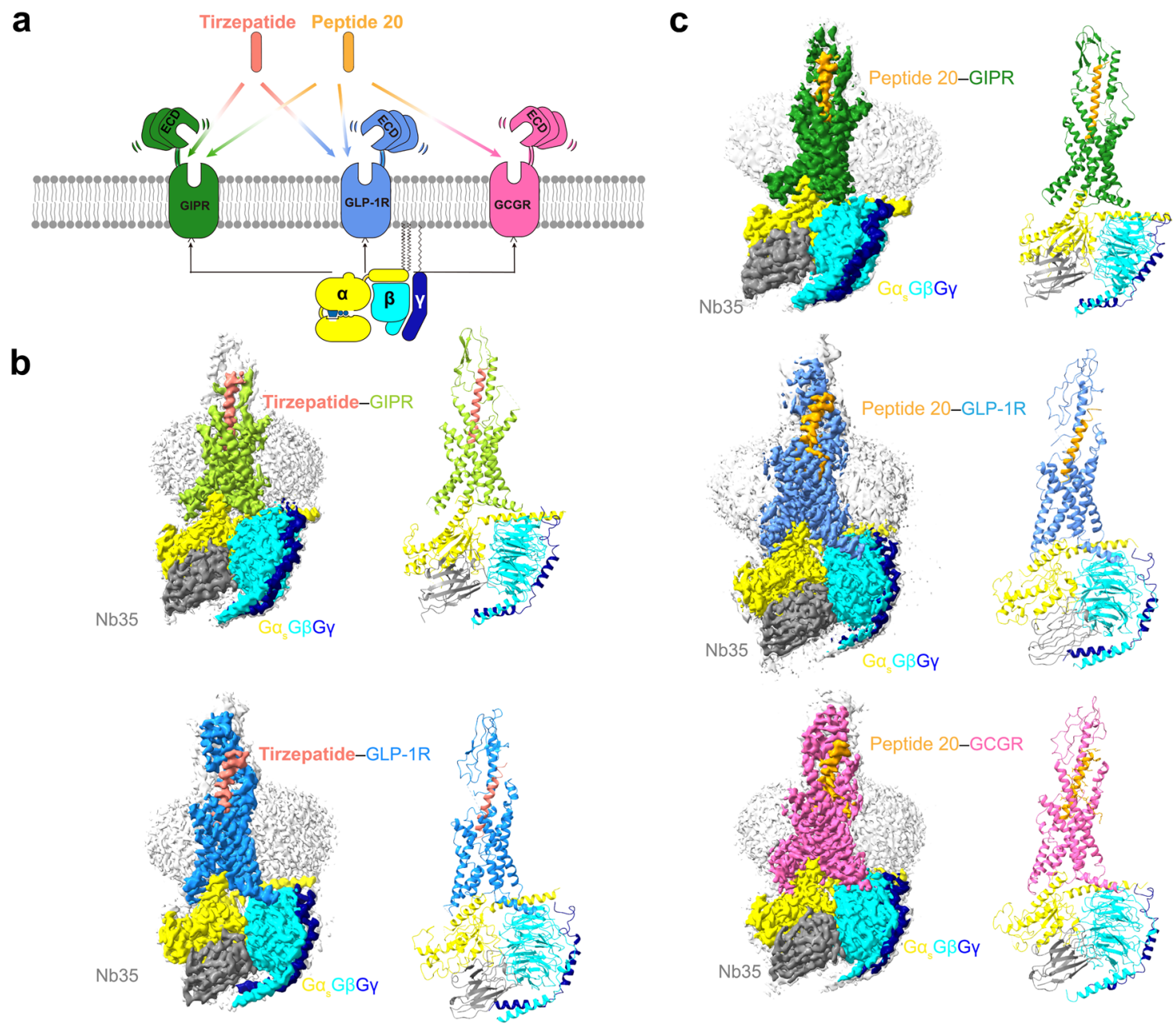

Fig. 1 Cryo-EM structures of tirzepatide and peptide 20-bound GIPR, GLP-1R and GCGR in complex with $\mathbf{G}_{\mathbf{s}}$ a Multi-targeting peptides tirzepatide and peptide 20 possess distinct combinatorial agonism at GIPR, GLP-1R and GCGR. b Cryo-EM maps (left) and structural models (right) of tirzepatide-bound GIPR (top) and GLP-1R (bottom) in complex with $\mathrm{G}_{\mathrm{s}}$. The sharpened cryo-EM density map at the 0.243 threshold shown as light gray surface indicates a micelle diameter of $10 \mathrm{~nm}$. The colored cryo-EM density map is shown at the 0.424 threshold. The tirzepatide is shown in salmon, GIPR in yellow green, GLP-1R in dodger blue, $G \alpha_{s}$ in yellow, $G \beta$ subunit in cyan, $G \gamma$ subunit in navy blue and Nb35 in gray. c Cryo-EM maps (left) and structural models (right) of peptide 20-bound GIPR (top), GLP-1R (middle) and GCGR (bottom) in complex with $\mathrm{G}_{\mathrm{s}}$. The sharpened cryo-EM density map at the 0.228 threshold shown as light gray surface indicates a micelle diameter of $11 \mathrm{~nm}$. The colored cryo-EM density map is shown at the 0.576 threshold. The peptide 20 is shown in orange, GIPR in forest green, GLP-1R in blue, GCGR in hot pink, G $\alpha_{\mathrm{s}}$ in yellow, G $\beta$ subunit in cyan, G $\gamma$ subunit in navy blue and Nb35 in gray.

solvent-accessible and of limited contact with GLP-1R, allowing them to employ distinct amino acids from GLP-1 without altering GLP-1R signaling profiles. As a comparison, superimposing either GIP or GCG with GLP-1 analogs suggest that they have potential steric clashes with ECL1 of GLP-1R via $\mathrm{H}_{1} 8^{\mathrm{P}}$ of GIP and $\mathrm{R} 18^{\mathrm{P}}$ of GCG. Two residues with shorter side chains $\left(\mathrm{I}^{\mathrm{P}}\right.$ and $\left.\mathrm{A}^{\mathrm{P}} \mathrm{P}\right)$ in GIP further weakened its binding to GLP-1R, consistent with the distinct cross-reactivity features of GIP and GCG with GLP-1 $\mathrm{R}^{5,6}$.

Superimposing the structures of GCGR- $\mathrm{G}_{\mathrm{s}}$ bound by $\mathrm{GCG}^{4}$, peptide 15 (GLP-1R and GCGR dual agonist) ${ }^{28}$ and peptide 20 reveals that these three peptides adopt a similar binding pose: a single continuous helix that penetrates into the TMD core through their N-terminal halves (residues 1 to 15 ), while the C-terminal halves (residues 16 to 30 ) are recognized by the ECD, ECL1 and TM1 (Fig. 2e). Given that both peptide 15 and peptide 20 are modified forms of GCG (differed by 7 residues), ligand recognition patterns are highly conserved across the three peptides except for a few positions. For example, by choosing alanine at position 18 instead of arginine in GCG, peptide 20 lost the cation-pi stacking with $\mathrm{W} 215^{\mathrm{ECL} 1}$ and hydrogen bond with Q204 $4^{\mathrm{ECL} 1}$, thereby allowing its outward movement toward ECL1 and leading to the formation of another hydrogen bond (D21 ${ }^{\mathrm{P}}-\mathrm{I} 206^{\mathrm{ECL} 1}$ ) (Fig. 2f). Probably due to the lack of complementary interacting residues, superimposing GIP or GLP-1 to GCG bound to GCGR shows that the aligned peptides reduced favorable contacts with GCGR compared to GCG by removing one hydrogen bond $\left(\mathrm{Y} 10^{\mathrm{P}}(\mathrm{GCG}) / \mathrm{Y} 10^{\mathrm{P}}(\mathrm{GIP}) / \mathrm{V} 16^{\mathrm{P}}(\mathrm{GLP}-1)-\mathrm{Q} 142^{1.40 \mathrm{~b}}(\mathrm{GCGR})\right)$ and pi-pi stacking $\left(\mathrm{Y}_{13} \mathrm{P}(\mathrm{GCG}) / \mathrm{A} 13^{\mathrm{P}}(\mathrm{GIP}) / \mathrm{Y} 19^{\mathrm{P}}(\mathrm{GLP}-1)-\mathrm{Y} 138^{1.36 \mathrm{~b}}\right.$ (GCGR)) and by repulsing the interaction between $\mathrm{Y}^{\mathrm{P}}(\mathrm{GIP})$ and $\mathrm{I} 235^{3.40 \mathrm{~b}}$ (GCGR). These observations receive the support of our current and previous functional data showing that both GIP and GLP-1 were unable to activate GCGR (Supplementary Table 6) $)^{5,6}$.

Collectively, the binding mode comparison of the three peptides bound by the same receptor demonstrates common structural features in ligand recognition and distinct conformational adaptability of GIPR, GLP-1R and GCGR in response to different agonist stimulation.

Recognition of tirzepatide. The tirzepatide-GIPR- $G_{s}$ and tirzepatide-GLP-1R-G $\mathrm{G}_{s}$ exhibit a similar peptide-receptor binding 

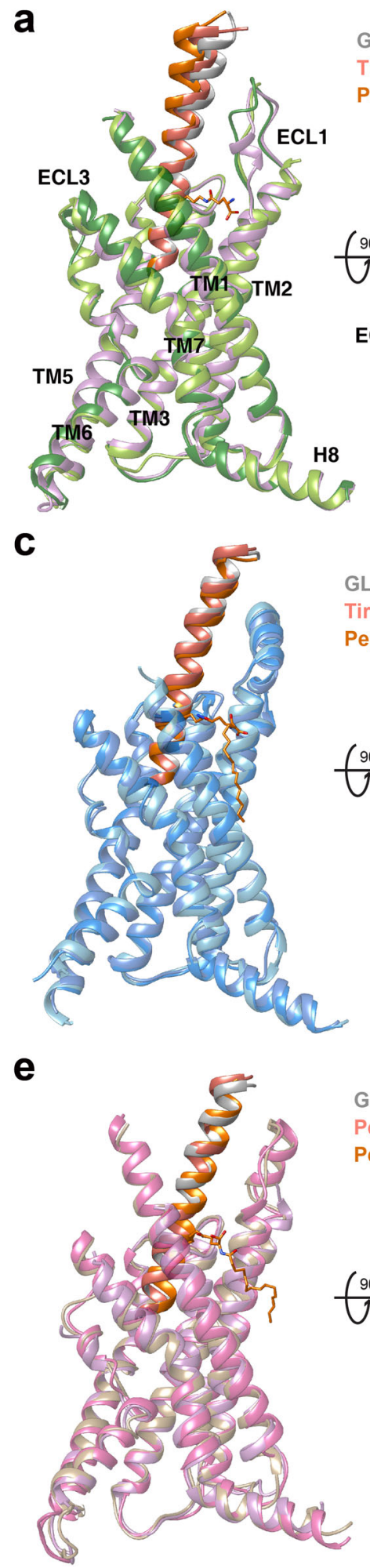

GIP-GIPR

Tirzepatide-GIPR

Peptide 20-GIPR

ECL3

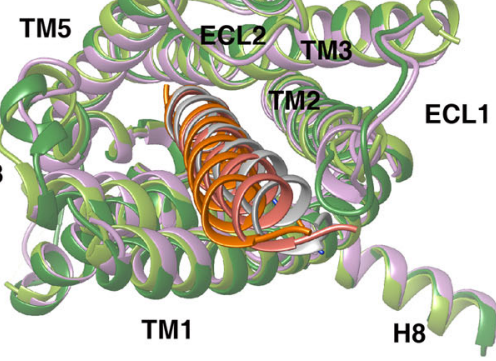

ECL1

$\int^{90^{\circ}}$

GLP-1-GLP-1R
Tirzepatide-GLP-1R
Peptide 20-GLP-1R

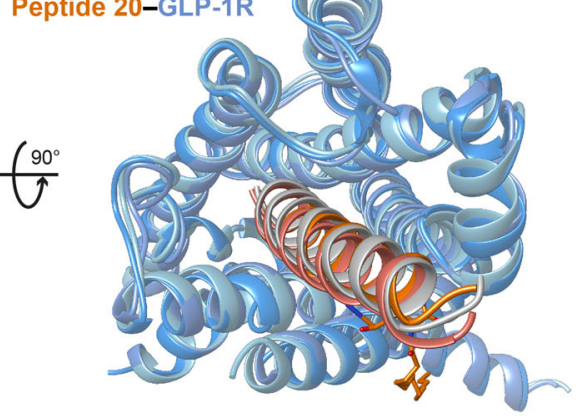

b

Salt bridges Hydrogen bonds Pi stacking Van der Waals interactions

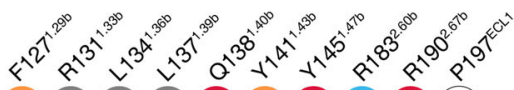

GIP

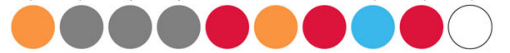

Tirzepatide 100000000

Peptide 20000000

GIP

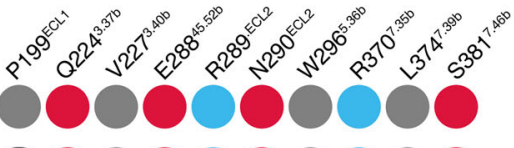

Tirzepatide 0000000000

Peptide $20 \bigcirc \bigcirc 00000$

d

GLP-1

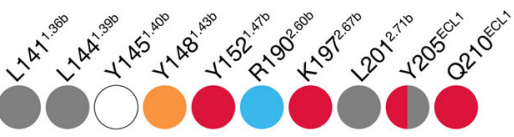

Tirzepatide 000000000

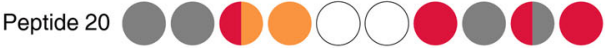

GLP-1

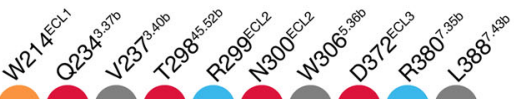

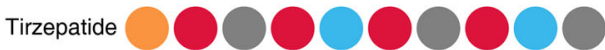

Peptide 20O000000000

f

GCG-GCGR

Peptide 15-GCGR

Peptide 20-GCGR

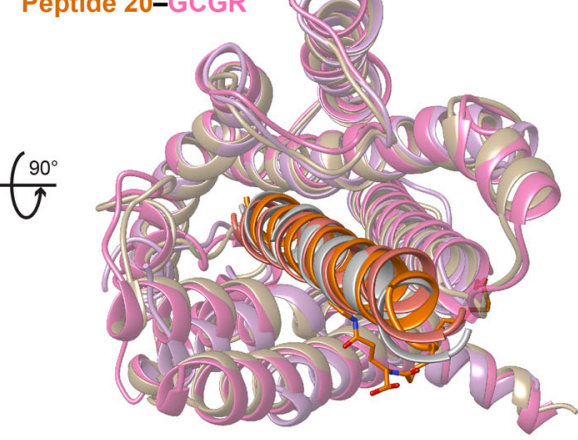

GCG

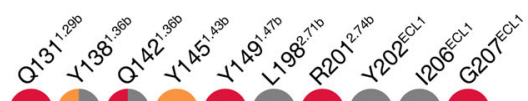

Peptide $15 \bigcirc 0000000$

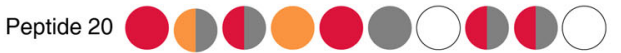

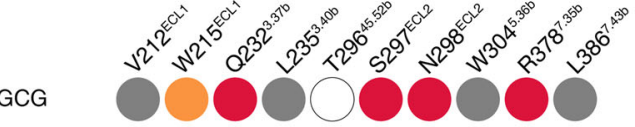

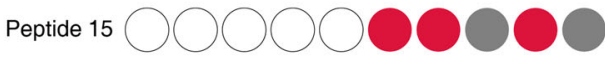

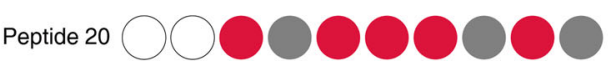

Fig. 2 Structural comparison of GIPR, GLP-1R and GCGR bound by mono-, dual and triple agonists. a Structural comparison of GIP-GIPR-G ${ }_{\mathrm{s}}^{25}$, tirzepatide-GIPR-G and peptide 20-GIPR-G. Receptor ECD and G protein are omitted for clarity. $\mathbf{b}$ Comparison of residue interactions employed by GIPR to recognize GIP, tirzepatide and peptide 20, described by fingerprint strings encoding different interaction types of the surrounding residues in each peptide. Color codes are listed on the top panel. Residues that show no interaction with ligands are displayed as white circles. c Structural comparison of GLP-1-GLP-1R-G ${ }_{s}^{27}$, tirzepatide-GLP-1R-G and peptide 20-GLP-1R-G. Receptor ECD and G protein are omitted for clarity. $\mathbf{d}$ Comparison of residue interactions that GLP-1R employed to recognize GLP-1, tirzepatide and peptide 20, described by fingerprint strings encoding different interaction types of the surrounding residues in each peptide. e Structural comparison of GCG-GCGR-G ${ }_{s}^{4}$, peptide $15-G C G R-G_{s}{ }^{28}$ and peptide $20-G C G R-G_{s}$. Receptor ECD and $\mathrm{G}$ protein are omitted for clarity. $\mathbf{f}$ Comparison of residue interactions that GCGR employed to recognize GCG, peptide 15 and peptide 20 , described by fingerprint strings encoding different interaction types of the surrounding residues in each peptide. 

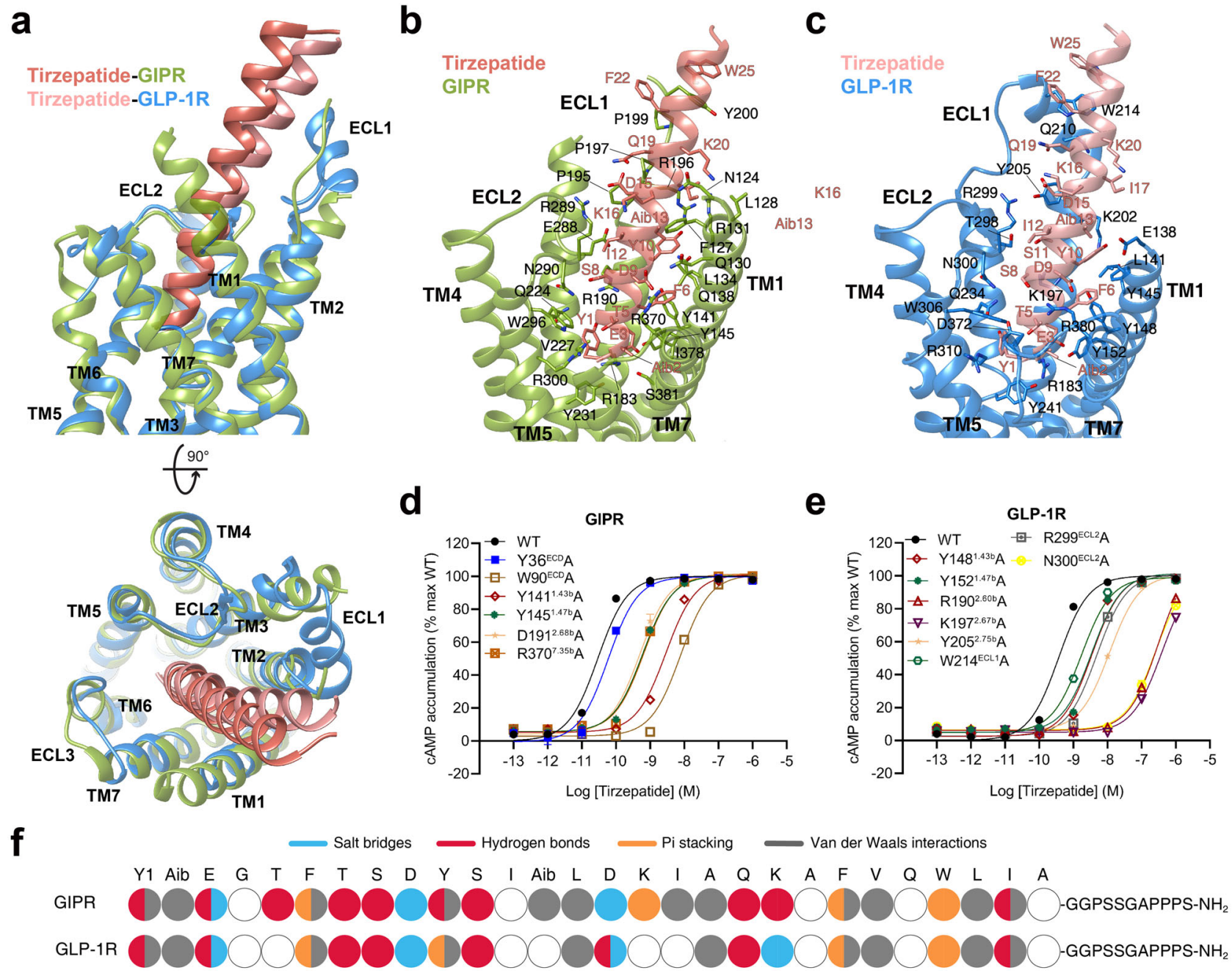

Fig. 3 Molecular recognition of tirzepatide by GIPR and GLP-1R. a Structural comparison of tirzepatide-GIPR-G and tirzepatide-GLP-1R-G . Receptor ECD and G protein are omitted for clarity. $\mathbf{b}$ Interactions between tirzepatide (salmon) and the TMD of GIPR (yellow green). Residues involved in interactions are shown as sticks. c Interactions between tirzepatide (light salmon) and the TMD of GLP-1R (dodger blue). Residues involved in interactions are shown as sticks. d-e Effects of receptor mutations on tirzepatide-induced cAMP accumulation. Data shown are means \pm S.E.M. of three independent experiments $(n=3)$ performed in quadruplicate. $\mathbf{f}$ The peptide recognition modes are described by fingerprint strings encoding different interaction types of the surrounding residues in each receptor. Residues that show no interaction with receptors are displayed as white circles. Color codes are listed on the top panel. WT, wild-type. Source data are provided as a Source Data file.

interface, where distinct structural features were observed at ECL1, ECL3 and the extracellular tips of TM1 and TM3 (Fig. 3a). GIPRbound tirzepatide is rotated by $8.3^{\circ}$ compared to that in complex with GLP-1R, such a movement shifted its $C$ terminus toward the TMD core by $5.2 \AA$ (measured by the Ca of $127^{\mathrm{P}}$ ). The N-terminal region of tirzepatide (residues 1 to 10 ) in GIPR and GLP-1R overlapped well with the formation of a network of extensive interactions with multiple conserved residues $\left(\mathrm{Y}^{1.43 \mathrm{~b}}, \mathrm{Y}^{1.47 \mathrm{~b}}\right.$, R190/K1972.67b, Q ${ }^{3.37 b}, \mathrm{~V}^{3.40 \mathrm{~b}}, \mathrm{~N} 290 / \mathrm{N} 300^{\mathrm{ECL} 2}, \mathrm{R}^{7.35 \mathrm{~b}}$ and I378/ L388 ${ }^{7.43 b}$ ) (Fig. 3b-e, Supplementary Tables 4, 7). Notably, the inward movement of GIPR R3005.40b contributed one hydrogen bond with $\mathrm{T}^{\mathrm{P}}$ (Fig. $3 \mathrm{~b}, \mathrm{f}$ ). The middle region of tirzepatide in GLP-1R was stabilized by the peptide-ECD-ECL1-ECL2 interface

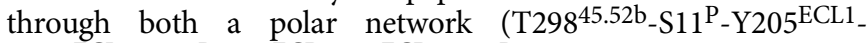
$\left.\mathrm{R} 299^{\mathrm{ECLL}}-\mathrm{D} 15^{\mathrm{P}}-\mathrm{L} 32^{\mathrm{ECD}}-\mathrm{S} 31^{\mathrm{ECD}}-\mathrm{Q} 19^{\mathrm{P}}\right)$ and a complementary nonpolar network with ECD (L32, V36, W39 and Y88), ECL1 (W214), TM1 (L141 ${ }^{1.36 b}$ ) and TM2 (L201 $\left.{ }^{2.71 b}\right)$ via $\mathrm{S}_{1}{ }^{\mathrm{P}}, \mathrm{Aib}^{\mathrm{B}} 3^{\mathrm{P}}$, $\mathrm{F} 22^{\mathrm{P}}, \mathrm{W} 25^{\mathrm{P}}$ and $\mathrm{L} 26^{\mathrm{P}}$ (Fig. 3c). As a comparison, the ECL1 of GIPR partially unwound with the presence of three proline residues $\left(\mathrm{P} 195^{\mathrm{ECL} 1}, \mathrm{P} 197^{\mathrm{ECL} 1}\right.$ and $\left.\mathrm{P} 199^{\mathrm{ECL} 1}\right)$, resulting in reduced interactions between ECL1 and tirzepatide compared to that in GLP-1R (Fig. 3b). However, the $\alpha$-helical extension in TM1 of GIPR provides additional residues for tirzepatide recognition including one hydrogen bond $\left(\mathrm{Y}_{10}^{\mathrm{P}}\right.$ and $\left.\mathrm{Q} 138^{1.40 \mathrm{~b}}\right)$ and a stacking interaction $\left(\mathrm{K}_{16} \mathrm{P}\right.$ and $\left.\mathrm{F} 127^{1.29 \mathrm{~b}}\right)$. These observations receive support of our mutagenesis studies by measuring both cAMP responses and receptor binding affinities (Fig. 3d, e and Supplementary Table 7). The acylation on $\mathrm{K}^{2} 0^{\mathrm{P}}$ by $\gamma \mathrm{Glu}$ - $2 \times \mathrm{OEG}$ linker and $\mathrm{C} 18$ fatty diacid moiety that enables enhanced binding to plasma albumin and extended the peptide half-life in vivo ${ }^{29}$ were not resolved in both structures, indicating a high conformational flexibility, in line with the recently published cryo-EM structure of semaglutide-bound GLP-1R $\mathrm{R}^{30}$ and our molecular dynamics (MD) simulation results (Supplementary Fig. 8a-c). Consistently, the non-acylated tirzepatide maintained high affinity and potency to both GLP-1R and GIPR (Supplementary Fig. 2f, g). Besides, GIPR has a relatively larger conformational difference between tirzepatide-bound and non-acylated tirzepatide-bound structures in most regions compared with GLP-1R (Supplementary Fig. 7c). 

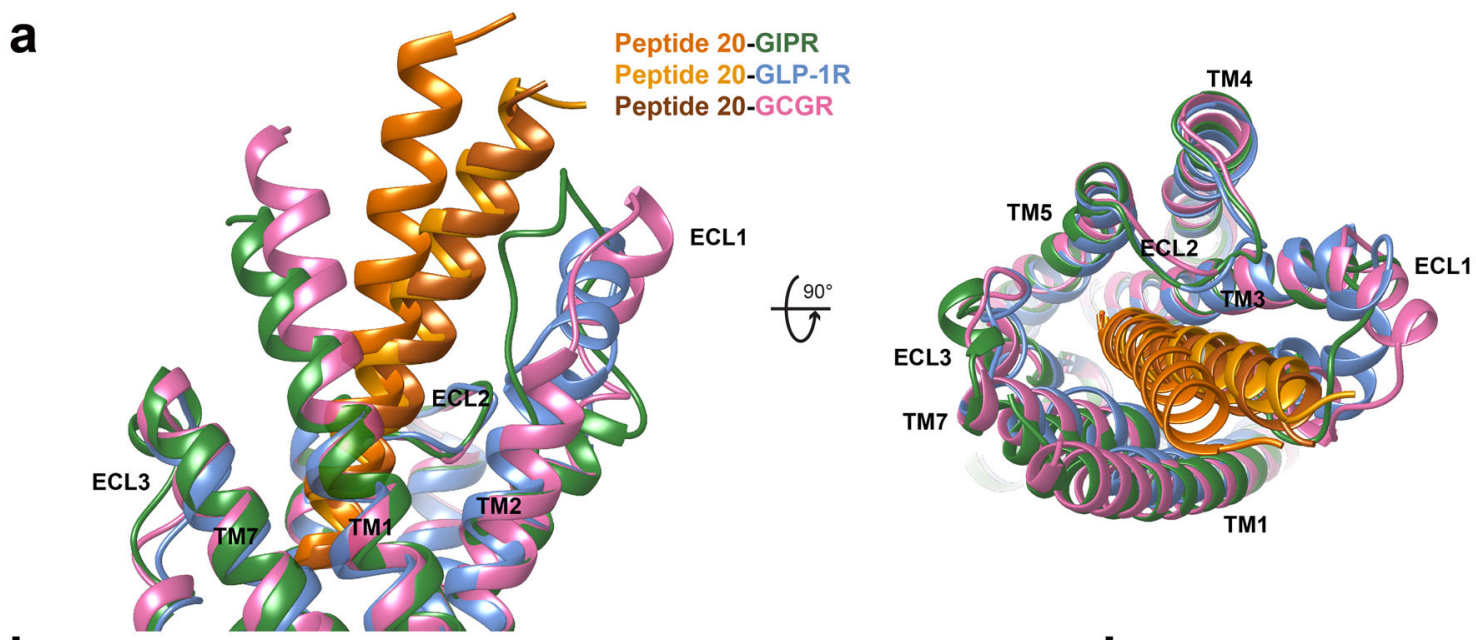

b
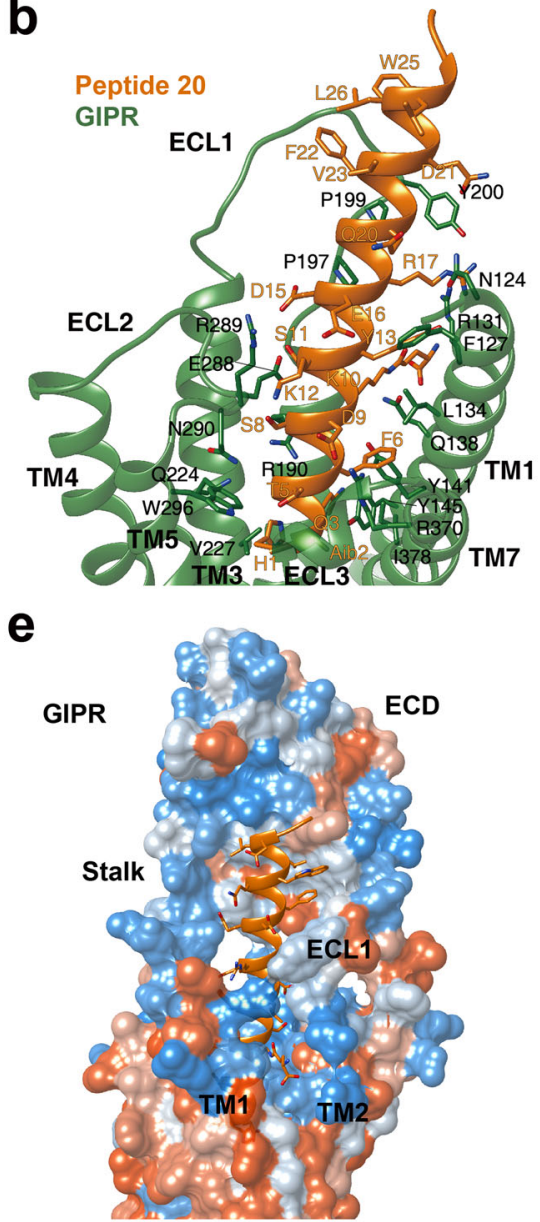

C

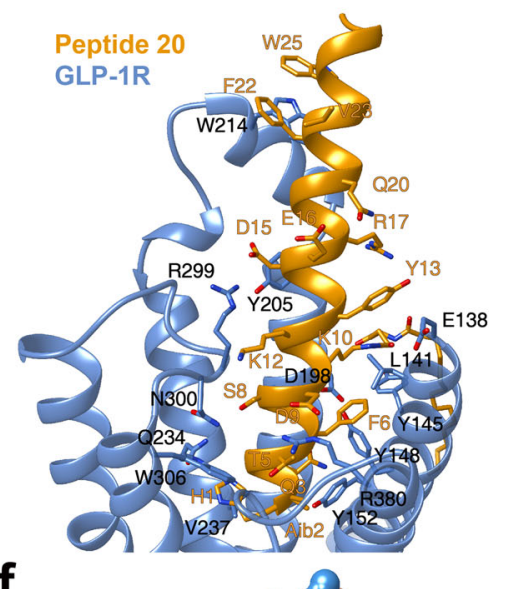

f

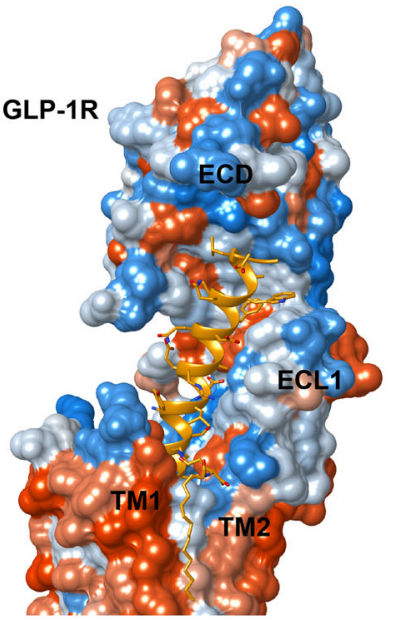

d

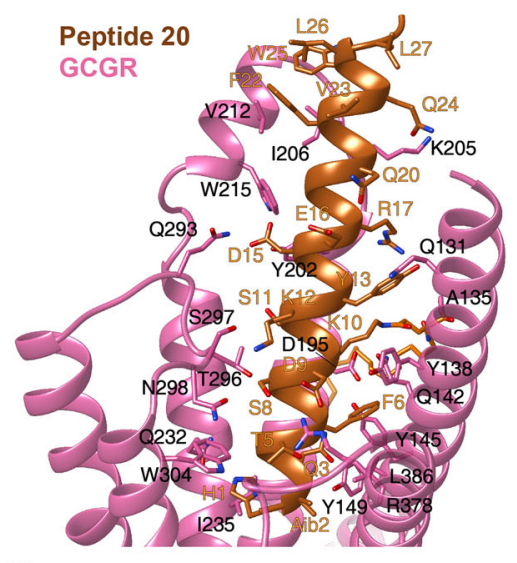

g

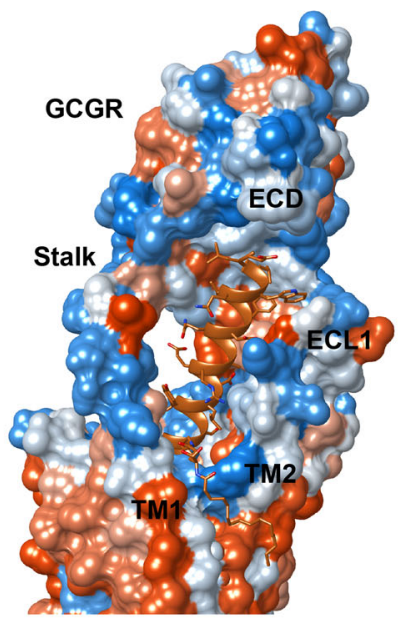

h

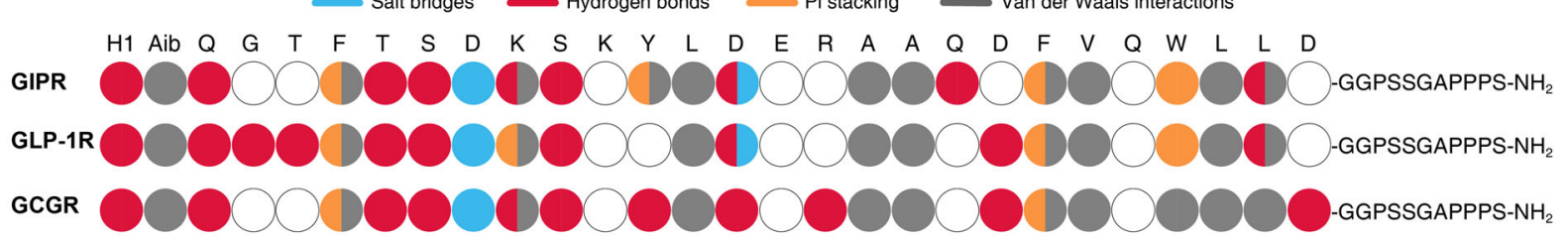

Peptide 20 recognition. Superimposition of the TMDs of GIPR, GLP-1R and GCGR bound by peptide 20 shows that the three receptors employed conserved residues in the lower half of the TMD pocket to recognize the well-overlapped peptide $\mathrm{N}$-terminal region (residues 1 to 11), while the peptide $\mathrm{C}$ terminus, engaged by ECL1, the $\mathrm{N}$-terminal $\alpha$-helix of ECD and the extracellular tip of TM1, displays receptor-specific positions and orientations (Fig. 4). Accompanying the inward movement of GIPR ECL1 by $6.4 \AA$ relative to that of GCGR (measured by Ca of G202 ${ }^{\mathrm{ECL} 1}$ in GIPR and G207 ${ }^{\mathrm{ECL} 1}$ in GCGR), the C terminus of peptide 20 bound by GIPR shifted toward the TMD core by $8.1 \AA$ (measured by $\mathrm{Ca}$ of $\mathrm{L}^{\mathrm{P}} \mathrm{P}^{\mathrm{P}}$ ) and consequently pushed the extracellular tip of 
Fig. 4 Molecular recognition of peptide 20 by GIPR, GLP-1R and GCGR. a Structural comparison of peptide 20-GIPR-G peptide 20-GCGR-G. Receptor ECD and G protein are omitted for clarity. b-d Interactions between peptide 20 and the TMDs of GIPR (forest green), GLP$1 R$ (blue), and GCGR (hot pink). Residues involved in interactions are shown as sticks. e- $\mathbf{g}$ Surface representations of the receptor for each of the peptidereceptor complex, with the peptides shown as ribbon and sticks. The receptor is shown in surface representation and colored from dodger blue for the most hydrophilic region, to white, to orange red for the most hydrophobic region. $\mathbf{h}$ The peptide recognition modes are described by fingerprint strings encoding different interaction types of the surrounding residues in each receptor. Color codes are listed on the top panel. Residues that show no interaction with receptors are displayed as white circles.

TM1 towards TM7 by $2.8 \AA$ (measured by Ca of the residues at $1.29 \mathrm{~b})$. ECL1 and ECD of the three receptors coincidently constructed a complementary binding groove for the entrance of the $\mathrm{C}$ terminus of peptide 20 through multiple hydrophobic residues $\left(\mathrm{A} 19^{\mathrm{P}}, \mathrm{F} 22^{\mathrm{P}}, \mathrm{V} 23^{\mathrm{P}}, \mathrm{W} 25^{\mathrm{P}}, \mathrm{L} 26^{\mathrm{P}}\right.$ and $\left.\mathrm{L} 27^{\mathrm{P}}\right)$. However, several additional interactions were observed in GLP-1R $\left(\mathrm{S}_{1} 1^{\mathrm{P}}-\mathrm{Y} 205^{\mathrm{ECL} 1}\right.$ and $\left.\mathrm{D} 21^{\mathrm{P}}-\mathrm{Q} 210^{\mathrm{ECL} 1}\right)$ and GCGR (D15 ${ }^{\mathrm{P}}-\mathrm{Y} 202^{\mathrm{ECL} 1}$ and $\mathrm{D} 21^{\mathrm{P}}$ I206 ${ }^{\mathrm{ECL} 1}$ ), but not in GIPR (Fig. 4b-h, Supplementary Table 5). Measurements of cAMP responses and ligand binding affinities in mutated GIP, GLP-1 and GCG receptors yielded similar results (Supplementary Fig. 9 and Supplementary Tables 8, 9).

Notably, strong cryo-EM densities were observed in the crevices between TM1 and TM2 of the three complexes (Fig. $5 \mathrm{a}-\mathrm{c}$ ). They were connected to the side chain end of $\mathrm{K} 10^{\mathrm{P}}$ of peptide 20, allowing unambiguous assignment of the binding sites of lipidated $\mathrm{K} 10^{\mathrm{P}}$ with a 16 -carbon palmitic acid through a $\gamma$-carboxylate spacer (Fig. $5 \mathrm{~d}-\mathrm{f}$ ). Such a modification on $\mathrm{K} 10^{\mathrm{P}}$ greatly stabilized the peptide binding through extensive contacts with both receptors and lipid membrane. For GCGR, the lipidated $\mathrm{K}_{10} \mathrm{P}$ contributed three hydrogen bonds (with S1391.37b, Q1421.40b and R1992.72b), extensive hydrophobic contacts with V143 $1.41 \mathrm{~b}, \mathrm{~T} 146^{1.44 \mathrm{~b}}$, L192 $2.65 \mathrm{~b}, \mathrm{~V} 193^{2.66 \mathrm{~b}}$, and membrane lipids where the 16-carbon palmitic chain surrounded (Fig. 5d-f). Removal of these contacts by GCGR triple mutant (Q142A + D195A + R199A) markedly reduced peptide 20 potency by 93 -fold (Fig. 5g). For GLP-1R, the $\gamma$-carboxylate spacer formed two hydrogen bonds (with Y1451.40b and $\mathrm{D} 198^{2.68 \mathrm{~b}}$ ), and the 16-carbon palmitic chain terminus dropped down along TM1 with the formation of multiple strong hydrophobic interactions with $\mathrm{I} 146^{1.41 \mathrm{~b}}$, T1491.44b V1501.45b, A153 $1.48 \mathrm{~b}$ and L154 $1.49 \mathrm{~b}$. Similar phenomenon was also observed in GIPR. Consistently, regardless of the initial positions of palmitate either located in the membrane as seen in the cryo-EM structure or placed outside of the membrane, our MD simulations found that the $\gamma$-carboxylate spacer stably inserted into the TM1TM2 cleft and the 16-carbon palmitic chain is deeply buried in the receptor-lipid interface, contributing multiple strong contacts to stabilize the complexes (Supplementary Fig. 10). The importance of $\mathrm{K}_{10} \mathrm{P}$ lipidation receives the support of our structure-activity relationship study where peptide 20 without $\mathrm{K} 10^{\mathrm{P}}$ lipidation reduced the receptor-mediated cAMP accumulation by 1,288 -fold and 759 -fold for GIPR and GCGR in the presence of $0.1 \%$ bovine serum albumin (BSA), respectively, but inappreciably influenced that of GLP-1R (Fig. 5h, Supplementary Table 10). Similar phenomenon was observed in the presence of $0.1 \%$ casein. These results suggest that specific modification of peptide is equally significant to sequence optimization in terms of demonstration of a desired polypharmacology of a unimolecular dual or triple agonist.

Receptor activation. Despite the existence of unique structural features among the ligand-binding pockets of GIPR, GLP-1R and GCGR, both tirzepatide and peptide 20 triggered receptor conformational changes similar to that induced by GLP-1 or GCG ${ }^{4,27}$ and distinct from the inactive or apo GLP-1R and GCGR structures (Supplementary Fig. 11) 31,32 . Compared to the inactive
GCGR, the extracellular tip of TM7 in peptide 20-bound GCGR moved outward by $5.1 \AA$ (measured by Ca atom of L377 ${ }^{7.34 b}$ ) and the $a$-helical structure of the extracellular half of TM6 was partially unwounded. In the intracellular side, a sharp kink located in the conserved Pro ${ }^{6.47 b}-\mathrm{X}-\mathrm{X}-\mathrm{Gly} \mathrm{C}^{6.50 \mathrm{~b}}$ motif pivoted the intracellular tip of TM6 to move outwards by $19.3 \AA$ (measured by Ca atom of $\mathrm{K} 344^{6.35 \mathrm{~b}}$ ), slightly higher than that seen with the GCG-GCGR-G $(17.7 \AA)^{4}$. This, in conjunction with the movement of TM5 towards TM6, opened up the cytoplasmic face of GCGR to accommodate $G$ protein coupling. Similar conformational change was also observed in the tirzepatide-GIPR-G,

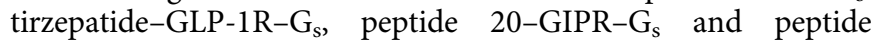
20-GLP-1R-G s complexes, compared to peptide-free apo GLP$1 \mathrm{R}$ structure ${ }^{31}$. At the residue level, signaling initiation by either peptide 20, tirzepatide or endogenous peptide hormones rendered a common arrangement of residue contacts for the three receptors, including the reorganization of the central polar network that located just below the peptide binding site, opening of the hydrophobic packing to favor the formation of the TM6 kink at the PXXG motif and the rearrangement of two polar networks (HETX motif and TM2-6-7-helix 8) at the cytoplasmic face ${ }^{33,34}$.

G protein coupling. Comparison of the two tirzepatide- and three peptide 20 -bound $\mathrm{GPCR}-\mathrm{G}_{\mathrm{s}}$ complex structures with that of other class B1 GPCR family members reveals a high similarity in the $G$ protein binding interface, suggesting a common mechanism for $G_{s}$ engagement ${ }^{4,33,35-38}$ (Fig. 6a). These complexes are anchored by the a 5 helix of $\mathrm{Ga}_{\mathrm{s}}$, which fits to the cytoplasmic cavity formed by TMs 2, 3, 5, 6, 7 and intracellular loop 1 (ICL1). Besides, H8 contributes several polar interactions with the $\mathrm{G} \beta$ subunit. There are some receptor- and ligand-specific structural features displayed by ICL2. For peptide 20-bound GCGR, its ICL2 moved downward and made extensive polar and nonpolar contacts with the binding groove formed by the $\alpha \mathrm{N}$ helix, $\beta 1$ strand and $\alpha 5$ helix of $G \alpha_{s}$, resulting in an ICL2-Ga interface area of $799 \AA^{2}$, significantly larger than that of GLP-1R $\left(396 \AA^{2}\right)$ or GIPR $\left(416 \AA^{2}\right.$ ) (Fig. 6b). Different from the dipped down side chain conformation observed in GLP-1-bound GLP$1 \mathrm{R}^{27}, \mathrm{~F} 257^{3.60 \mathrm{~b}}$ in the peptide $20-\mathrm{GLP}-1 \mathrm{R}-\mathrm{G}_{\mathrm{s}}$ complex rotated its side chain upwards (Fig. 6c). Furthermore, E262 ${ }^{\mathrm{ICL} 2}$ was reoriented $\sim 90^{\circ}$ from an outside facing position to a position pointing to $\mathrm{Ga}_{\mathrm{s}}$, thus introducing a hydrogen bond with Q35 $\mathrm{GaHN}(\mathrm{GaHN}$ means the $a \mathrm{~N}$ helix of $\mathrm{Ga}$ ) (Fig. $6 \mathrm{~d}$ ). Similar $\mathrm{G}$ protein interface was also observed in the tirzepatide-bound GLP-1R except for the orientation of E262 ICL2 that is closer to that of GLP-1. In the case of peptide 20- and tirzepatide-bound GIPR complexes, the side chain of E253 ${ }^{\mathrm{ICL} 2}$ contributed one salt bridge with $\mathrm{K} 34^{\mathrm{GaHN}}$, not observed in the peptide 20-bound GLP-1R and GCGR complexes (Fig. 6e).

Efficacy superiority. The superior therapeutic efficacy of tirzepatide over approved selective GLP-1 analogs was reported recently ${ }^{17,39}$, whereas the outcome of clinical trials on peptide 20 is not available in the literature. The five high-resolution cryo-EM structures reported here, together with abundant structural and 
a

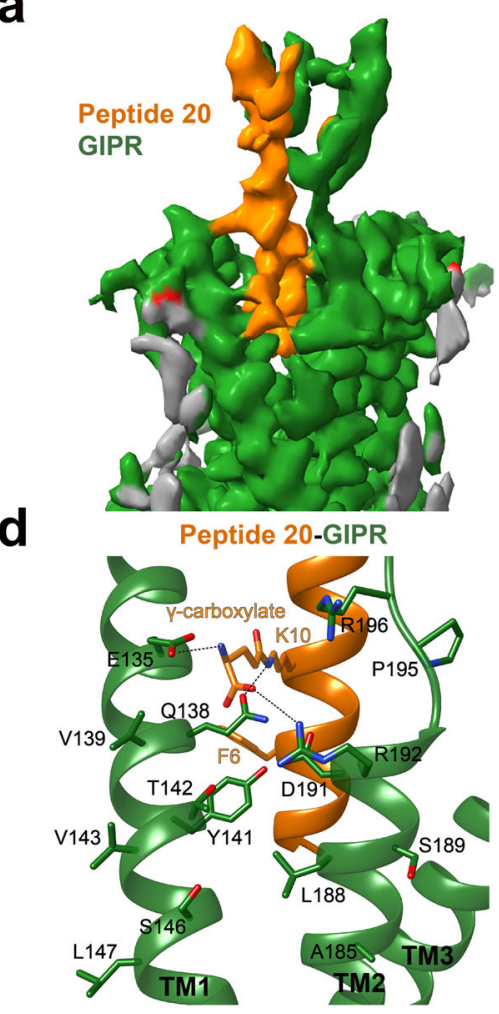

b

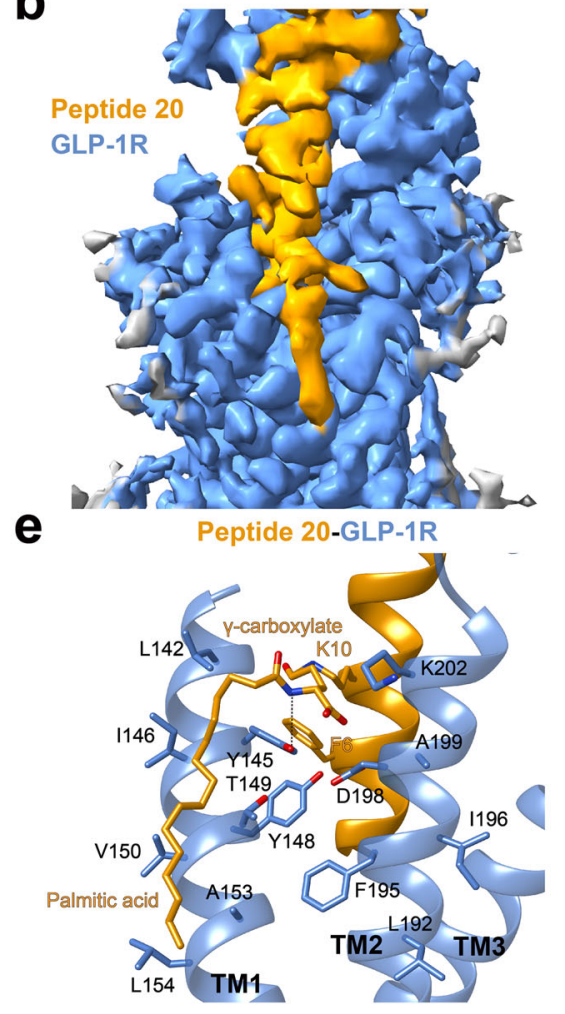

C
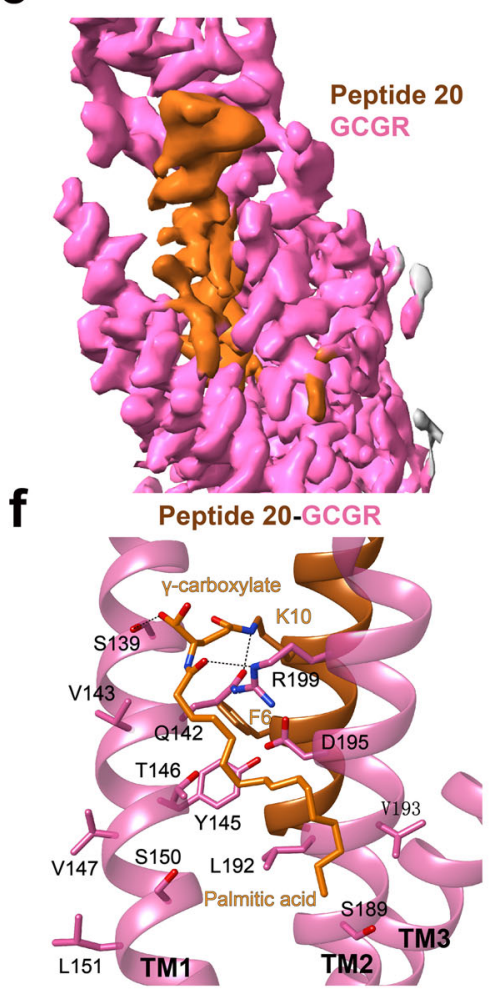
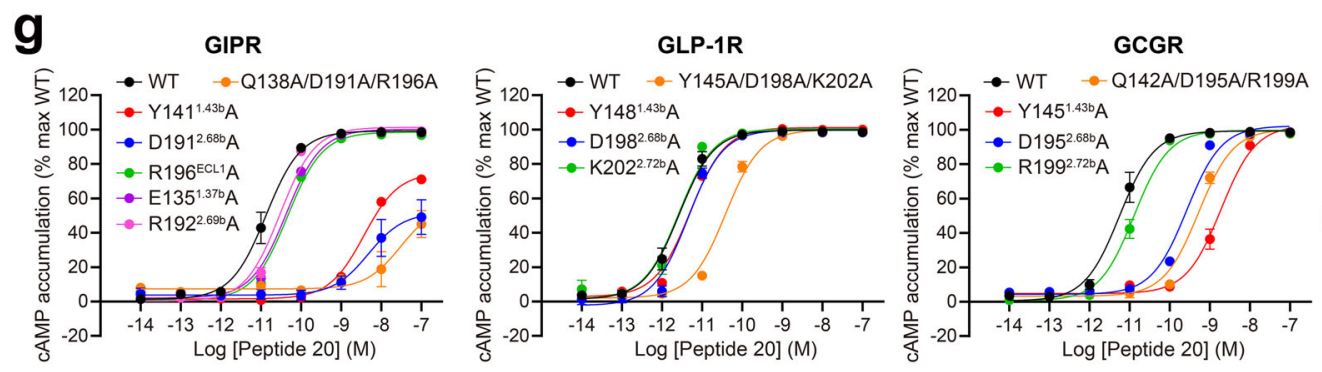

h

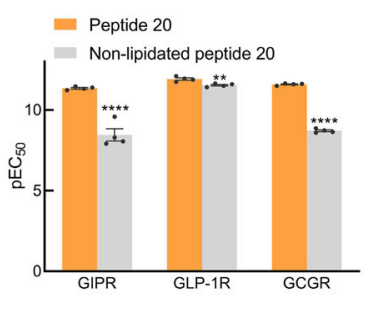

Fig. 5 Structural and functional feature of lipidated K10P of peptide 20. a-c Close-up views of the crevices between TM1 and TM2 displayed by cryo-EM maps of peptide 20-bound GIPR a, GLP-1R b, and GCGR c. Continuous electron densities connected to K10 in peptide 20 were observed in the three peptide 20-bound receptor- $G_{s}$ complexes. $\mathbf{d}$-f Interactions between lipidated K10P and the TM1-TM2 crevice of GIPR d, GLP-1R e, and GCGR f, with interacting residues shown in sticks. Hydrogen bonds are shown with dashed lines. $\mathbf{g}$ Effects of receptor mutations on peptide 20-induced cAMP accumulation. Data shown are means \pm S.E.M. of at least three independent experiments $(n=3-9)$ performed in quadruplicate. $\mathbf{h}$ Effects of K10 lipidation on peptide 20 -induced cAMP accumulation. The bar graph represents the average $\mathrm{pEC} \mathrm{C}_{50}$ (that is, $-\log \mathrm{EC}_{50}$ ) and data are presented as means $\pm \mathrm{S}$.E.M. of four independent experiments $(n=4)$ performed in quadruplicate. Statistically significant differences were determined with a two-tailed Student's t test. ${ }^{\star \star} P<0.01$ and ${ }^{\star \star \star \star} P<0.0001$. WT, wild-type. Source data are provided as a Source Data file.

pharmacological data of monospecific peptides documented previously $4,25,27,30,40$, provide us an excellent opportunity to analyze the molecular basis of the superior clinical efficacy presented by multi-targeting agonists.

Semaglutide and tirzepatide share two common substitutions, one is Aib at the second $\mathrm{N}$-terminal residue and the other is acylated lysine at the $20^{\text {th }} \mathrm{N}$-terminal residue by $\mathrm{C} 18$ diacid via a $\gamma$ Glu- $2 \times$ OEG linker, to reduce degradation by dipeptidyl peptidase-4 (DPP-4) and to prolong their half-lives by enhanced binding to plasma albumin (Fig. $7 \mathrm{a})^{41}$. Besides, there is only one residue in semaglutide $\left(\mathrm{R} 34^{\mathrm{P}}\right)$ that is different from GLP-1 but does neither form any interaction with GLP- $1 R^{30}$ nor affect receptor binding and signaling ${ }^{29}$. However, tirzepatide has 14 unique amino acids (engineered from the GIP sequence) and an amidated exenatide-like $C$ terminus as opposed to GLP-1 which allow the peptide to possess a GIPR binding ability equivalent to GIP(1-42) and to steadily interact with GLP-1R with a reduced potency compared to GLP-1 ${ }^{27}$ (Fig. 2a-d). Like GLP-1, semaglutide is not able to bind or activate GIPR. These findings were confirmed by GIPR or GLP-1R mediated cAMP accumulation assays (Fig. $7 \mathrm{~b}-\mathrm{c})^{39}$. Of note is that tirzepatide was previously reported to cause biased signaling at GLP-1R in favor of cAMP response over $\beta$-arrestin recruitment in the presence of $0.1 \%$ casein $^{39}$, which was confirmed in the present study showing that non-acylated tirzepatide elicited better cAMP responses $\left(\mathrm{EC}_{50}=\right.$ $0.90 \mathrm{pM}$ for $0.1 \%$ casein and $4.66 \mathrm{pM}$ for $0.1 \%$ BSA) than that of tirzepatide $\left(\mathrm{EC}_{50}=3.05 \mathrm{pM}\right.$ for $0.1 \%$ casein and $103.10 \mathrm{pM}$ for $0.1 \%$ BSA) (Supplementary Table 11) highlighting the impact of acylation on their pharmacological properties. The combined activation of GIPR and GLP-1R by tirzepatide not only improved both glucose-dependent insulin secretion and glucose tolerance in mice ${ }^{15}$, but also showed significantly better efficacy than semaglutide and dulaglutide with regard to glucose control and weight loss 16,17 . 


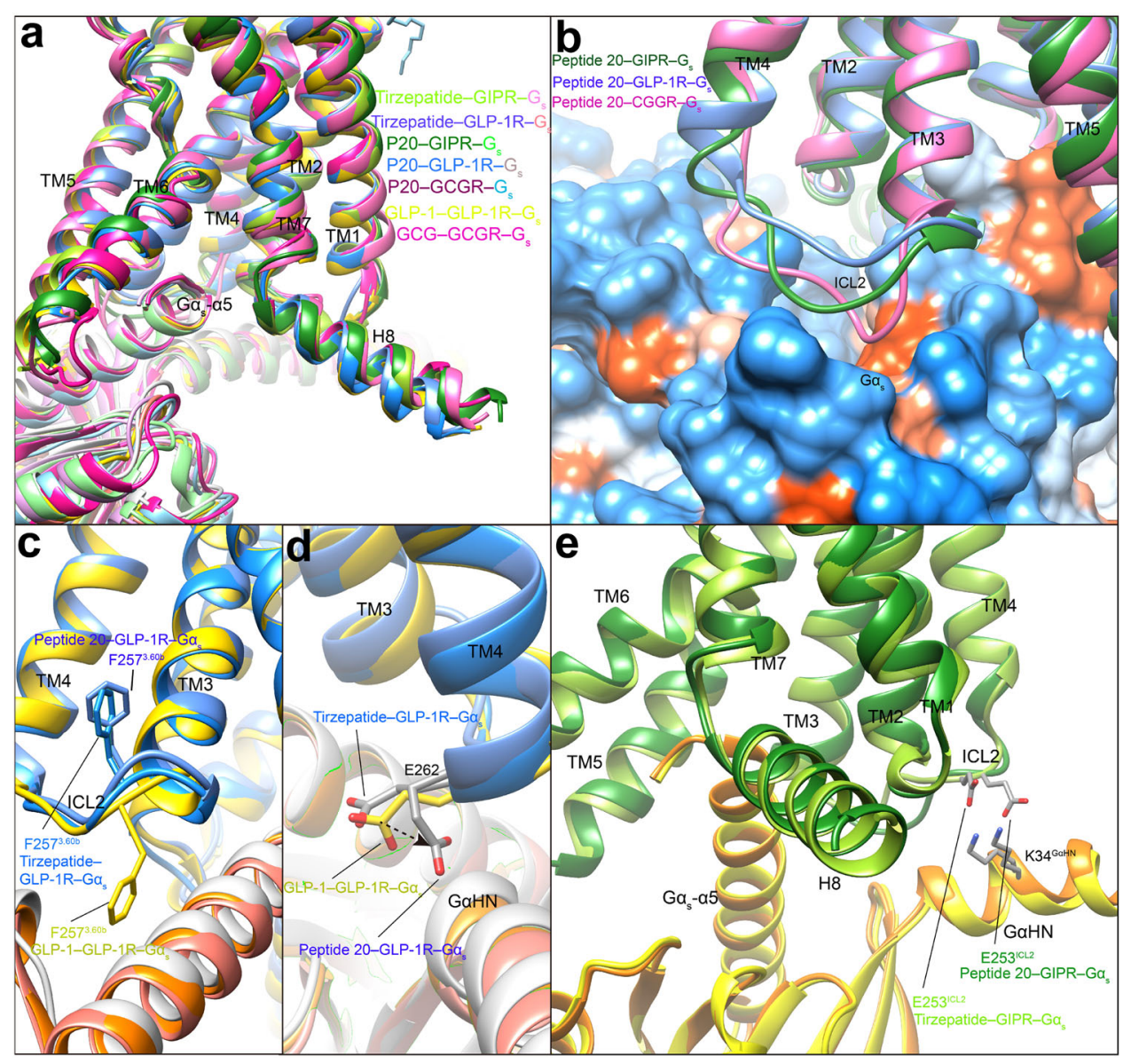

Fig. 6 G protein coupling of multi-targeting agonist-bound GIPR, GLP-1R and GCGR. a Comparison of G protein coupling among GIPR, GLP-1R and $\mathrm{GCGR} 4,25,27$. The $G \alpha_{s} \alpha 5$-helix of the $G \alpha_{s}$ Ras-like domain inserts into an intracellular crevice of receptor's TMD. The receptors and $G$ proteins are colored as the labels. b Comparison of ICL2 conformation in the peptide 20-bound GIPR, GCGR and GLP-1R. c Comparison of F2573.60b conformation in the GLP-1R bound by GLP-1, tirzepatide and peptide 20. d Comparison of E262 ${ }^{\mathrm{ICL} 2}$ conformation in the GLP-1R bound by GLP-1, tirzepatide and peptide 20 . e Comparison of E253 ICL2 conformation in the GIPR bound by tirzepatide and peptide 20. Residues involved in interactions are shown as sticks. Polar interactions are shown as black dashed lines.

It is known that peptide 20 potently reversed metabolic disorders in rodent models of obesity and diabetes, characteristic of increased energy expenditure and elevated circulating FGF21 levels as a result of GCGR agonism ${ }^{22,23}$. Meanwhile, peptide 20 causes a biased signaling at GIPR and GCGR in favor of cAMP accumulation over $\beta$-arrestin 2 recruitment, with no significant biased influence on GLP-1R signaling ${ }^{5,6}$. Both the $\mathrm{N}$ terminus and $\mathrm{C}$ terminus of peptide 20 play important roles in its multiplexed pharmacological actions. The $\mathrm{N}$ terminus (the first 11 residues) that is highly conserved across GIP, GLP-1 and GCG interacts with the lower half of the TMD pocket of the three receptors consisting of conserved residues such as $\mathrm{L} / \mathrm{Y}^{1.36 \mathrm{~b}}$ (hydrophobic with $\mathrm{K} 10^{\mathrm{P}}$ ), Q/ $\mathrm{Y}^{1.40 \mathrm{~b}}$ (hydrogen bond with $\mathrm{K} 10^{\mathrm{P}}$ ), $\mathrm{Y}^{1.43 \mathrm{~b}}$ (stacking with $\mathrm{F}^{\mathrm{P}}$ ), $\mathrm{Y}^{1.47 \mathrm{~b}}$ (hydrogen bond with $\mathrm{Q}^{\mathrm{P}}$ ), $\mathrm{Q}^{3.37 \mathrm{~b}}$ (hydrogen bond with $\mathrm{H}^{\mathrm{P}}$ ), ECL2 (hydrogen bond with $\mathrm{S}^{\mathrm{P}}$ ), $\mathrm{R}^{7.35 \mathrm{~b}}$ (salt bridge with $\mathrm{D}^{\mathrm{P}}$ ), $\mathrm{I} / \mathrm{L}^{7.43 \mathrm{~b}}$ (hydrophobic with $\mathrm{Aib}^{\mathrm{P}}{ }^{\mathrm{P}}$ ) and $\mathrm{L}^{7.43 \mathrm{~b}}$ (hydrophobic with F6 ${ }^{\mathrm{P}}$ ) (Figs. 2, 4b-d, 7a) ${ }^{42,43}$. A similar approach was applied to the design of peptide 20 's $\mathrm{C}$ terminus that occupies the hydrophobic binding groove of ECD, with residues $\left(\mathrm{A} 19^{\mathrm{P}}, \mathrm{F} 22^{\mathrm{P}}, \mathrm{V} 23^{\mathrm{P}}, \mathrm{W} 25^{\mathrm{P}}, \mathrm{L} 26^{\mathrm{P}}\right.$ and $\left.\mathrm{L} 27^{\mathrm{P}}\right)$ adopted from GIP, GLP-1 and GCG (Figs. 4e-g, 7a) ${ }^{42,43}$. To accommodate the upper half of the TMD pocket formed by ECL1 and the extracellular tips of TM1 and TM2 that diversified in both sequence and conformation across the three receptors, peptide 20 employs distinct residues to recognize specific region of a given receptor (Fig. 4h). For GIPR whose ECL1 was loosely compacted by peptide 20 , three residues $\left(\mathrm{Y}_{13}^{\mathrm{P}}-\mathrm{L} 14^{\mathrm{P}}-\mathrm{D} 15^{\mathrm{P}}\right)$ strengthened the peptide-binding interface by forming a hydrogen bond with F127 $1.29 \mathrm{~b}$ and a salt bridge with R289 ${ }^{\mathrm{ECL} 2}$, significantly stronger than that observed in GLP-1R and GCGR. Alternatively, another three residues $\left(\mathrm{D} 21^{\mathrm{P}}-\mathrm{F} 22^{\mathrm{P}}-\mathrm{W} 25^{\mathrm{P}}\right)$ compacted well with the ordered ECL1 of GLP-1R via a hydrogen bond with Q210 $\mathrm{ECL1}$ and packing with W214 $4^{\mathrm{ECL} 1}$. Two hydrogen bonds (D15 $\mathrm{P}_{\text {- }}$ $\mathrm{Y} 202^{2.75 b}$ and $\mathrm{R} 17^{\mathrm{P}}-\mathrm{Y} 202^{2.75 b}$ ) were only seen in GCGR.

The most impressive structural feature of peptide 20 is the lipidated $\mathrm{K} 10^{\mathrm{P}}$ by a 16-carbon palmitic acid through a $\gamma$ carboxylate spacer, which perfectly inserted into the TM1-TM2 crevice and made extensive contacts with both receptors and lipid membrane to stabilize the binding poses (Fig. 5). These observations suggest a combined mechanism of action for peptide 20 that uses conserved residues for ligand recognition and specific residues to induce conformational changes unique to each receptor, leading to a highly potent and balanced multitargeting agonist for GIPR, GLP-1R and GCGR with a cAMP signaling profile similar to that of GIP, GLP-1 and GCG (Fig. $7 b)^{22}$.

\section{Discussion}

Due to the central roles exerted by the three metabolically related peptide hormone receptors (GIPR, GLP-1R and GCGR) in the management of T2DM and obesity, the concept of combinatorial 
a

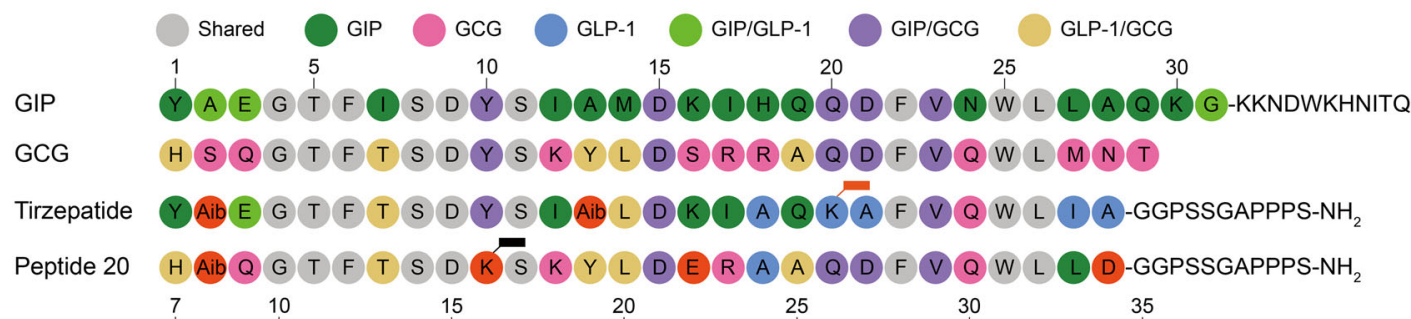

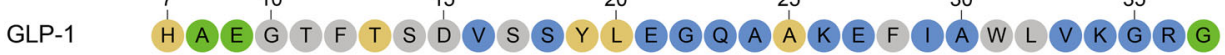
Semaglutide H Aib E G T F TS DVS Y LE G A A K E I A W L VR GR G

b
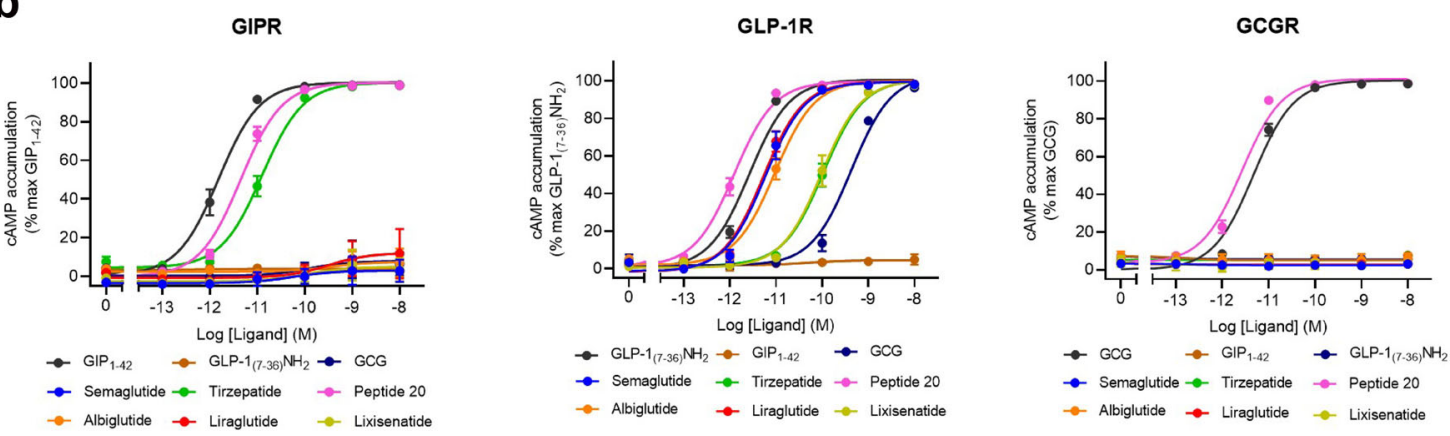

C

\begin{tabular}{|c|c|c|c|}
\hline Peptide & GIPR (pIC $50 \pm$ S.E.M.) & GLP-1R (pIC $5_{50} \pm$ S.E.M.) & GCGR ( pIC $_{50} \pm$ S.E.M.) \\
\hline GIP & $7.88 \pm 0.04$ & N.B. & N.B. \\
\hline GLP-1 & N.B. & $8.11 \pm 0.10$ & N.B. \\
\hline GCG & N.B. & $6.15 \pm 2.37$ & $6.67 \pm 0.18$ \\
\hline Semaglutide & N.B. & $8.34 \pm 0.17$ & N.B. \\
\hline Tirzepatide & $7.50 \pm 0.10$ & $8.09 \pm 0.16$ & N.B. \\
\hline Peptide 20 & $8.18 \pm 0.13$ & $8.47 \pm 0.23$ & $6.97 \pm 0.18$ \\
\hline Albiglutide & N.B. & $8.42 \pm 0.09$ & N.B. \\
\hline Liraglutide & N.B. & $8.50 \pm 0.11$ & N.B. \\
\hline Lixisenatide & N.B. & $8.80 \pm 0.13$ & N.B. \\
\hline
\end{tabular}

N.B., no binding was detected.

Fig. 7 Structure-basis of receptor selectivity demonstrated by tirzepatide, peptide $\mathbf{2 0}$ and GLP-1 analogs. a Amino acid sequences of endogenous agonists, multi-targeting agonists and approved GLP-1 analogs including semaglutide. Residues are colored according to sequence conservation among GIP, GLP-1 and GCG. Aib, aminoisobutyric acid. Semaglutide and tirzepatide are conjugated by a C20 fatty diacid moiety via a linker connected to the lysine

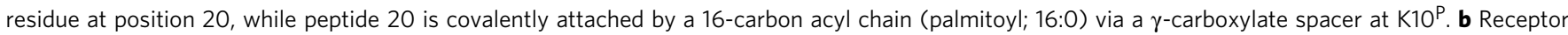
signaling profiles of endogenous agonists, multi-targeting agonists and approved drug GLP-1 analogs including semaglutide. Data shown are means \pm S.E.M. of four independent experiments $(n=4)$ performed in quadruplicate. Source data are provided as a Source Data file. c Receptor binding profiles of endogenous agonists, multi-targeting agonists and approved GLP-1 analogs. Data shown are means \pm S.E.M. of three independent experiments ( $n=3$ ) performed in duplicate.

agonism or polypharmacology to synergize metabolic actions and maximize therapeutic benefits has been explored in the past decade with remarkable preclinical and clinical achievements. The 3-dimensional structures of GCGR, GLP-1R and GIPR solved previously helped us better understand the molecular basis of ligand recognition and receptor activation of these important class B1 GPCRs ${ }^{25,32,44-46}$. In this paper, we report five cryo-EM structures of two well-recognized multi-targeting agonists (tirzepatide and peptide 20) in complex with individual receptors and $\mathrm{G}_{\mathrm{s}}$ proteins. The structural basis of the superior clinical efficacy of tirzepatide relative to monospecific agonists such as semaglutide and the triagonist peptide 20 is elucidated. Our results provide a near-atomic level visualization of the molecular action of multitargeting agonists on three cognate receptors and offer valuable information for the design of better drugs to combat metabolic diseases.

Superimpositions of the two tirzepatide- and three peptide 20bound structures to the three receptors bound by the endogenous ligands (GIP, GLP-1 and GCG) showed that the five peptides all adopt a single continuous helix, with the well-overlapped $\mathrm{N}$ terminus penetrating to the TMD core stabilized by conserved interactions, while the $\mathrm{C}$ terminus anchors the ECD, ECL1 and ECL2 in a receptor- and ligand-specific manner. With the presence of three proline residues (P195 ${ }^{\mathrm{ECL} 1}, \mathrm{P} 197^{\mathrm{ECL} 1}$ and P199ECL1), the ECL1 of GIPR presents a notable conformational adaptability in recognition of different agonists, a phenomenon that was not seen with that of GLP-1R and GCGR as their binding pockets exhibit less flexibility when recognizing the peptides through a combination of common segment that contributes to conserved interactions and distinct sequences that govern receptor selectivity. The distinct sequences that tirzepatide and peptide 20 employed, respectively, to recognize GIPR or GLP-1R are obviously different: the former was primarily based on the GIP sequence with engineered GLP-1 activity ${ }^{15}$, whereas the latter was derived from a GLP-1R/GCGR dual agonist in conjunction with GIP agonism ${ }^{22}$. Such a sequence and receptor binding divergence may consequently alter pharmacological and clinical outcomes. Clearly, distinct sequence and structural 
features of tirzepatide and peptide 20 allow them to exert combinatorial agonism at two or more receptors at the same time thereby maximize the benefit of polypharmacology and minimize the limitation of mono-targeting.

Both GIP and GLP-1 are released upon nutrient ingestion to promote insulin secretion by pancreatic $\beta$-cells. However, they have opposed effects on circulating GCG levels ${ }^{7,16}$. GIPR activation also has different roles in lipid metabolism from that of GLP-1 ${ }^{47}$. Maintenance of GCG action might be a key to the superior therapeutic efficacy of tirzepatide ${ }^{16,17,48}$. Structurally, the binding of tirzepatide to GIPR reshaped the ECL1 conformation relative to that of GIP, but made no change in the GLP-1R structure. As far as peptide 20 is concerned, the peptide binding pocket of both GLP-1R and GCGR closely resembled that of GLP-1 and GCG bound structures, where notable conformational change was only observed in the ECL1 of GIPR. These differences in structural plasticity or rigidity among the three receptors give clues to further optimize multi-targeting agonists using complementary amino acids to target common regions of individual receptors and distinct sequences to confer receptor selectivity.

Unlike tirzepatide that retains GCG function via counteracting that of GLP-1 through activation of GIPR, causing a glucagonotropic action by stimulating GCG release in states of hypoglycemia ${ }^{7,49}$, peptide 20 is capable of activating GCGR directly. Consistent with the effects of GCGR in increasing lipolysis and thermogenesis besides elevating blood glucose levels, preclinical studies have found that peptide 20 improved energy metabolism and hepatic lipid handling without exacerbating preexisting hyperglycemia 22 . Peptide 20 was developed through a series of optimizing processes based on GCGR agonism in dietinduced obese mice, concluding that the ideal metabolic benefits of triagonism predominantly depend on fine-tuning the GCG component ${ }^{22}$. The structures reveal that lipidation at K10 of peptide 20 allows the hydrophobic acyl tail to interact with the TMD region of all three receptors, providing a fresh clue for peptidic ligand design. Previous studies on optimizing the peptide sequences towards potent dual or triple agonists provide important information on the combinatorial agonism. For example, the subtle modification on the third $\mathrm{N}$-terminal residue of peptide 20 showed diversified effects on its triagonism, highlighting the complexity of peptide-induced signal transduction. From the perspective of precision medicines, combinatorial agonism might be precisely designed to reflect pharmacological profiles of individual receptors such that diabetic patients at different disease stages could be prescribed with different multi-targeting agonists to take personalized therapeutic advantages.

\section{Methods}

Cell lines. Spodoptera frugiperda 9 (Sf9) (Invitrogen) and High Five ${ }^{\mathrm{mix}}$ insect cells (Expression Systems) were cultured in ESF 921 serum-free medium (Expression Systems) at $27^{\circ} \mathrm{C}$ and $120 \mathrm{rpm}$. Human embryonic kidney 293 cells containing SV40 large T-antigen (HEK293T) were cultured in DMEM (Gibco) supplemented with $10 \%(\mathrm{v} / \mathrm{v})$ fetal bovine serum (FBS, Gibco), $1 \mathrm{mM}$ sodium pyruvate (Gibco) and 100 units $/ \mathrm{mL}$ penicillin and $100 \mu \mathrm{g} / \mathrm{mL}$ streptomycin at $37^{\circ} \mathrm{C}$ in $5 \% \mathrm{CO}_{2}$. Chinese hamster ovary (CHO-K1) cells were cultured in F-12 (Gibco) containing $10 \% \mathrm{FBS}, 100 \mathrm{units} / \mathrm{mL}$ penicillin and $100 \mu \mathrm{g} / \mathrm{mL}$ streptomycin at $37^{\circ} \mathrm{C}$ in $5 \% \mathrm{CO}_{2}$. For cAMP and receptor expression assays, HEK293T cells were seeded into 6-well cell culture plates at a density of $7 \times 10^{5}$ cells per well. After overnight incubation, cells were transfected with GIPR, GLP-1R or GCGR construct using Lipofectamine 2000 or Lipofectamine 3000 transfection reagent (Invitrogen). For whole-cell binding assay, $\mathrm{CHO}-\mathrm{K} 1$ cells were seeded into $96-$ well fibronectin-treated cell culture plates at a density of $3 \times 10^{4}$ cells per well. After overnight incubation, cells were transfected with GIPR, GLP-1R or GCGR construct using Lipofectamine 2000 transfection reagent (Invitrogen). Following $24 \mathrm{~h}$ culturing, the transfected cells were ready for use.

Construct. The human GIPR DNA (Genewiz) with one mutation (T345F) was cloned into the pFastBac vector (Invitrogen) with its native signal peptide replaced by the haemagglutinin (HA) signal peptide. A BRIL fusion protein was added at the
$\mathrm{N}$-terminal of the ECD with a TEV protease site and 2GSA linker between them C-terminal 45 amino acids (Q422-C466) of the receptor were truncated. LgBiT was added at the end of helix 8 with a 15-amino acid (15AA) polypeptide linker in between, followed by a TEV protease cleavage site and an OMBP-MBP tag. A dominant-negative bovine $\mathrm{Ga}_{\mathrm{s}}\left(\mathrm{DNGa}_{\mathrm{s}}\right)$ construct with 9 mutations (S54N, G226A, E268A, N271K, K274D, R280K, T284D, I285T and A366S) $)^{50,51}$ was used to help stabilize the tirzepatide-GIPR- $\mathrm{G}_{\mathrm{s}}$ complex. Meanwhile, a $\mathrm{DNG} \alpha_{\mathrm{s}}$ construct with 8 mutations (S54N, G226A, E268A, N271K, K274D, R280K, T284D and I285T) was used to help stabilize the peptide $20-G I P R-G_{s}$ complex $^{38,51}$. Rat G $\beta 1$ was cloned with a C-terminal SmBiT34 (peptide 86 or HiBiT, Promega) connected with a 15AA polypeptide linker. The modified rat $G \beta 1$ and bovine $G \gamma 2$ were both cloned into a pFastBac vector. The construct and various mutants of human GIPR were cloned into pcDNA3.1 vector for cAMP accumulation and whole-cell binding assays.

The human GLP-1R was modified with its native signal sequence (M1-P23) replaced by the HA signal peptide to facilitate receptor expression. To obtain a GLP-1R-G $\mathrm{G}_{\mathrm{s}}$ complex with good homogeneity and stability, we used the NanoBiT tethering strategy, in which the C terminus of GLP-1R was directly attached to LgBiT subunit followed by a TEV protease cleavage site and a double MBP tag. Rat $\mathrm{G} \beta 1$ was the same as the construct used in the GIPR structure determination. The $\mathrm{Ga}_{\mathrm{s}}\left(\mathrm{DNG} \alpha_{\mathrm{s}}\right.$ with 9 mutations) used to stabilize the tirzepatide-GLP-1R-G complex was the same as that employed for the tirzepatide-GIPR- $\mathrm{G}_{\mathrm{s}}$ complex. A dominant-negative human $\mathrm{Ga}_{\mathrm{s}}\left(\mathrm{DNGa} \mathrm{s}_{\mathrm{s}}\right.$ ) with 8 mutations (S54N, G226A, E268A $\mathrm{N} 271 \mathrm{~K}, \mathrm{~K} 274 \mathrm{D}, \mathrm{R} 280 \mathrm{~K}, \mathrm{~T} 284 \mathrm{D}$, and I285T) was generated as previously described to limit $\mathrm{G}$ protein dissociation ${ }^{51}$. The constructs were cloned into both pcDNA3.1 and $\mathrm{pFastBac}$ vectors for functional assays in mammalian cells and protein expression in insect cells, respectively. Other constructs including the full-length and various mutants of human GLP-1R were cloned into pcDNA3.1 vector for cAMP accumulation and whole-cell binding assays.

The human GCGR gene was cloned into pFastBac1 vector with GP64 promoter at the $\mathrm{N}$ terminus to enhance the protein yield. Forty-five residues (H433-F477) were truncated at the $\mathrm{C}$ terminus to improve the thermostability and an affinity tag, HPC4 tag, was added to the C terminus (GP64-HA-GCGR-GSGS linker-HPC4). $\mathrm{Ga}_{\mathrm{s}}\left(\mathrm{DNG} \alpha_{\mathrm{s}}\right.$ with 8 mutations) was modified as above to stabilize the interaction with $\beta \gamma$ subunits. The rat $\mathrm{G} \beta 1$ and bovine $\mathrm{G} \gamma 2$ were used in the structure determination.

Additionally, we used an engineered $\mathrm{G}_{\mathrm{s}}$ (mini- $\mathrm{G}_{\mathrm{s}}$ ) protein to stabilize the nonacylated tirzepatide (the side chain was removed at C20) bound GIPR or GLP-1R as described previously ${ }^{52}$

Protein expression. Baculoviruses containing the above complex constructs were prepared by the Bac-to-Bac system (Invitrogen). For the tirzepatide-GIPR- $\mathrm{G}_{\mathrm{s}}$ and non-acylated tirzepatide-GIPR-mini- $\mathrm{G}_{\mathrm{s}}$ complexes, GIPR and $\mathrm{DNG} \alpha_{\mathrm{s}}$ or mini-G heterotrimer were co-expressed in High Five ${ }^{m}$ cells. Briefly, insect cells were grown in ESF 921 culture medium (Expression Systems) to a density of $3.2 \times 10^{6}$ cells $/ \mathrm{mL}$ The cells were then infected with BRIL-TEV-2GSA-GIPR(22-421)T345F-15AALgBiT-TEV-OMBP-MBP, DNGa $\alpha_{\mathrm{s}}$ or mini- $\mathrm{G}_{\mathrm{s}}$, G $\beta 1$-peptide 86 and G 2 , respectively, at a ratio of 1:4:4:4. For the peptide $20-\mathrm{GIPR}-\mathrm{G}_{\mathrm{s}}$ complex, GIPR and $\mathrm{G}_{\mathrm{s}}$ heterotrimer were co-expressed in High Five ${ }^{\mathrm{m} t}$ cells grown in ESF 921 culture medium (Expression Systems) to a density of $3.2 \times 10^{6}$ cells $/ \mathrm{mL}$. The cells were then infected with BRIL-TEV-2GSA-GIPR(22-421)T345F-15AA-LgBiT-TEVOMBP-MBP, $D N G \alpha_{s}, G \beta 1$-peptide 86 and $G \gamma 2$, respectively, at a ratio of 1:3:3:3. After $48 \mathrm{~h}$ incubation at $27^{\circ} \mathrm{C}$, the cells were collected by centrifugation and stored at $-80^{\circ} \mathrm{C}$ until use.

The GLP-1R-LgBiT-2MBP, $\mathrm{DNGa}_{\mathrm{s}}$ or mini- $\mathrm{G}_{\mathrm{s}}$, G $\beta 1$-peptide 86 and $\mathrm{G} \gamma 2$ were co-expressed at multiplicity of infection (MOI) ratio of 1:1:1:1 by infecting $S f f$ cells at a density of $3.0 \times 10^{6}$ cells $/ \mathrm{mL}$. Other operations are the same as GIPR.

The GCGR construct, DNGa $\alpha_{s}$ and G $\beta 1$ and G $\gamma 2$ were co-expressed in High Five $^{\text {twt }}$ cells and infected with four separate baculoviruses at a ratio of 4:1:1:1. Other operations are the same as GIPR.

Nb35 expression and purification. Nanobody $35(\mathrm{Nb} 35)$ with a $6 \times$ his tag at the C-terminal was expressed in the periplasm of E. coli BL21 (DE3) cells. Briefly, Nb35 target gene was transformed in the bacterium and amplified in TB culture medium with $100 \mu \mathrm{g} / \mathrm{mL}$ ampicillin, $2 \mathrm{mM} \mathrm{MgCl}, 0.1 \%$ (w/v) glucose at $37^{\circ} \mathrm{C}, 180 \mathrm{rpm}$. When OD600 reached $0.7-1.2,1 \mathrm{mM}$ IPTG was added to induce expression followed by overnight incubation at $28^{\circ} \mathrm{C}$. The cell pellet was then collected under $4^{\circ} \mathrm{C}$ and stored at $-80^{\circ} \mathrm{C}$. Nb35 was purified by size-exclusion chromatography (SEC) using a HiLoad 16/600 Superdex 75 column (GE Healthcare) with running buffer containing $20 \mathrm{mM}$ HEPES, $100 \mathrm{mM} \mathrm{NaCl}$, pH 7.4. Fractions of Nb35 were concentrated to $\sim 2 \mathrm{mg} / \mathrm{mL}$ and quickly frozen in the liquid nitrogen with $10 \%$ glycerol and stored in $-80^{\circ} \mathrm{C}$.

Complex formation and purification. Tirzepatide, non-acylated tirzepatide and peptide 20 were chemically synthesized in GL Biochem with a purity of $95.89 \%$, $95.56 \%$ and $96.46 \%$, respectively. For the tirzepatide-GIPR- $\mathrm{G}_{\mathrm{s}}$ complex, cell pellets were lysed in a buffer containing $20 \mathrm{mM}$ HEPES, $100 \mathrm{mM} \mathrm{NaCl}, \mathrm{pH} 7.4,10 \mathrm{mM}$ $\mathrm{MgCl}_{2}, 1 \mathrm{mM} \mathrm{MnCl} 2$ and $10 \%$ glycerol supplemented with protease inhibitor cocktail, EDTA-free (TragetMol). Cell membranes were then collected by 
ultracentrifugation at $4{ }^{\circ} \mathrm{C}, 90,000 \mathrm{~g}$ for $35 \mathrm{~min}$. A buffer consisting of $20 \mathrm{mM}$ HEPES, $100 \mathrm{mM} \mathrm{NaCl}, \mathrm{pH} 7.4,10 \mathrm{mM} \mathrm{MgCl}_{2}, 1 \mathrm{mM} \mathrm{MnCl}_{2}$ and $10 \%$ glycerol was used to re-suspend the collected membranes. To assemble the GIPR-G $\mathrm{G}_{\mathrm{s}}$ complex, $15 \mu \mathrm{M}$ tirzepatide (GL Biochem) was added to the preparation accompanied by $100 \mu \mathrm{M}$ TCEP, $25 \mathrm{mU} / \mathrm{mL}$ apyrase (Sigma-Aldrich), $20 \mu \mathrm{g} / \mathrm{mL} \mathrm{Nb35}$ and $100 \mathrm{U}$ salt active nuclease (Sigma-Aldrich) supplemented with protease inhibitor cocktail for $1.5 \mathrm{~h}$ incubation at room temperature (RT). The membrane was then solubilized with $0.5 \%(\mathrm{w} / \mathrm{v})$ lauryl maltose neopentylglycol (LMNG, Anatrace) and $0.1 \%(\mathrm{w} / \mathrm{v})$ cholesterol hemisuccinate (CHS, Anatrace) with additional $2 \mu \mathrm{M}$ tirzepatide for $3 \mathrm{~h}$ at $4{ }^{\circ} \mathrm{C}$. The supernatant was isolated by centrifugation at $90,000 \mathrm{~g}$ for $35 \mathrm{~min}$ and the solubilized complex was incubated with amylose resin (NEB) for $2.5 \mathrm{~h}$ at $4{ }^{\circ} \mathrm{C}$. The resin was collected by centrifugation at $550 \mathrm{~g}$ and loaded onto a gravity flow column. The resin in the column was first washed with 5 column volumes (CVs) of buffer containing $20 \mathrm{mM}$ HEPES, pH 7.4, $100 \mathrm{mM} \mathrm{NaCl}, 10 \%$ (v/v) glycerol, $5 \mathrm{mM}$ $\mathrm{MgCl}_{2}, 1 \mathrm{mM} \mathrm{MnCl} 2,25 \mu \mathrm{M}$ TCEP, $5 \mu \mathrm{M}$ tirzepatide, $0.1 \%$ (w/v) LMNG and $0.02 \%(\mathrm{w} / \mathrm{v})$ CHS. After this, the resin was further washed with $25 \mathrm{CVs}$ of buffer containing $20 \mathrm{mM}$ HEPES, pH 7.4, $100 \mathrm{mM} \mathrm{NaCl}, 10 \%$ (v/v) glycerol, $5 \mathrm{mM}$ $\mathrm{MgCl}_{2}, 1 \mathrm{mM} \mathrm{MnCl} 2,25 \mu \mathrm{M}$ TCEP, $5 \mu \mathrm{M}$ tirzepatide, 0.03\% (w/v) LMNG, $0.01 \%$ $(\mathrm{w} / \mathrm{v})$ glyco-diosgenin (GDN, Anatrace) and $0.008 \%(\mathrm{w} / \mathrm{v}) \mathrm{CHS}$. The protein was then incubated with a buffer consisting of $20 \mathrm{mM}$ HEPES, pH 7.4, $100 \mathrm{mM} \mathrm{NaCl}$, $10 \%$ (v/v) glycerol, $5 \mathrm{mM} \mathrm{MgCl} 2,1 \mathrm{mM} \mathrm{MnCl} 2,25 \mu \mathrm{M}$ TCEP, $50 \mu \mathrm{M}$ tirzepatide, $20 \mu \mathrm{g} / \mathrm{mL} \mathrm{Nb35}, 0.03 \%$ (w/v) LMNG, $0.01 \%$ (w/v) GDN, 0.008\% (w/v) CHS and $30 \mu \mathrm{g} / \mathrm{mL}$ His-tagged TEV protease on the column overnight at $4{ }^{\circ} \mathrm{C}$. The flow through was collected and concentrated to $500 \mu \mathrm{L}$ using a $100 \mathrm{kDa}$ filter (Merck Millipore). SEC was performed by loading the protein onto Superose 6 Increase 10/ 300GL (GE Healthcare) column with running buffer containing $20 \mathrm{mM}$ HEPES, $\mathrm{pH}$ 7.4, $100 \mathrm{mM} \mathrm{NaCl}, 10 \mathrm{mM} \mathrm{MgCl}, 100 \mu \mathrm{M}$ TCEP, $5 \mu \mathrm{M}$ tirzepatide, $0.00075 \%$ (w/v) LMNG, 0.00025\% (w/v) GDN, 0.0002\% (w/v) CHS and $0.00025 \%$ digitonin (Anatrace). The tirzepatide-GIPR- $\mathrm{G}_{\mathrm{s}}$ complexes were collected and concentrated for cryo-EM analysis.

For the non-acylated tirzepatide-GIPR-mini- $\mathrm{G}_{\mathrm{s}}$ complex, the operations of the purification were the same as the tirzepatide-GIPR- $\mathrm{G}_{\mathrm{s}}$ complex, except that the peptide was replaced by the non-acylated tirzepatide. The complex samples were concentrated to $14-16 \mathrm{mg} / \mathrm{mL}$ for cryo-EM analysis.

For the tirzepatide-GLP-1R-G $\mathrm{G}_{\mathrm{s}}$ complex, cells were suspended in $20 \mathrm{mM}$ HEPES, $\mathrm{pH} 7.4,100 \mathrm{mM} \mathrm{NaCl}$ and $10 \%(\mathrm{v} / \mathrm{v})$ glycerol in the presence of protease inhibitor cocktail. Complex was formed by adding $10 \mathrm{mM} \mathrm{MgCl}_{2}, 1 \mathrm{mM} \mathrm{MnCl}_{2}, 50$ $\mathrm{mU} / \mathrm{mL}$ apyrase, $30 \mu \mathrm{M}$ tirzepatide, $100 \mu \mathrm{M}$ TCEP and $10 \mu \mathrm{g} / \mathrm{mL}$ Nb35 to the cell lysate and incubated at RT for $1.5 \mathrm{~h}$. Cell membranes were solubilized by adding $0.5 \%(\mathrm{w} / \mathrm{v})$ LMNG supplemented with $0.1 \%(\mathrm{w} / \mathrm{v}) \mathrm{CHS}$ at $4{ }^{\circ} \mathrm{C}$ for $2 \mathrm{~h}$, followed by centrifugation at $65,000 \mathrm{~g}$ for $30 \mathrm{~min}$ at $4{ }^{\circ} \mathrm{C}$. The supernatant was taken to bind with amylose resin for $2 \mathrm{~h}$ at $4^{\circ} \mathrm{C}$. After packing, the column was washed with buffer containing $20 \mathrm{mM}$ HEPES, pH 7.4, $100 \mathrm{mM} \mathrm{NaCl}, 10 \%(\mathrm{v} / \mathrm{v})$ glycerol, $5 \mu \mathrm{M}$ tirzepatide, $25 \mu \mathrm{M}$ TCEP, $5 \mathrm{mM} \mathrm{MgCl}, 1 \mathrm{mM} \mathrm{MnCl}_{2}, 0.1 \%$ (w/v) LMNG and $0.02 \%(\mathrm{w} / \mathrm{v})$ CHS first $(10 \mathrm{CVs})$, and then with decreased concentrations of detergents, $0.03 \%(\mathrm{w} / \mathrm{v})$ LMNG, $0.01 \%(\mathrm{w} / \mathrm{v}) \mathrm{GDN}$ and $0.006 \%(\mathrm{w} / \mathrm{v})$ CHS $(20$ CVs). TEV enzyme was added to the resin and kept at $4{ }^{\circ} \mathrm{C}$ overnight to remove the OMBP-MBP tag. The complex was eluted from the resin and concentrated to $500 \mu \mathrm{L}$ using a $100 \mathrm{kDa}$ MWCO Amicon Ultra Centrifugal Filter. SEC was carried out by loading the protein sample to Superdex 200 Increase 10/300GL (GE Healthcare) to obtain the monomer complex. The column was pre-equilibrated with $20 \mathrm{mM}$ HEPES, pH 7.4, $100 \mathrm{mM} \mathrm{NaCl}, 5 \mu \mathrm{M}$ tirzepatide, $100 \mu \mathrm{M}$ TCEP, $2 \mathrm{mM} \mathrm{MgCl}_{2}, 0.00075 \%$ (w/v) LMNG, $0.00025 \%$ (w/v) GDN, $0.00015 \%$ (w/v) CHS and $0.00025 \%$ digitonin.

For the non-acylated tirzepatide-GLP-1R-mini- $\mathrm{G}_{\mathrm{s}}$ complex, the operations of the purification were the same as the peptide $20-G L P-1 R-G_{s}$ complex, except that the peptide was replaced by the non-acylated tirzepatide, and the detergent of SEC running buffer was changed to $0.01 \%$ digitonin. The complex samples were concentrated to $16-18 \mathrm{mg} / \mathrm{mL}$ for cryo-EM analysis.

For the peptide $20-$ GIPR $-G_{s}$ complex, the operations of the purification was the same as the tirzepatide-GIPR- $\mathrm{G}_{\mathrm{s}}$ complex, except that the peptide was replaced by the peptide 20 . The complex samples were concentrated to $5-6 \mathrm{mg} / \mathrm{mL}$ for cryoEM analysis.

For the peptide $20-\mathrm{GLP}-1 \mathrm{R}-\mathrm{G}_{\mathrm{s}}$ complex, cell pellets were thawed and lysed in a buffer containing $20 \mathrm{mM}$ HEPES, pH 7.5, $100 \mathrm{mM} \mathrm{NaCl}, 10 \%$ (v/v) glycerol, $10 \mathrm{mM} \mathrm{MgCl}_{2}, 1 \mathrm{mM} \mathrm{MnCl}$ and $100 \mu \mathrm{M}$ TCEP supplemented with EDTA-free protease inhibitor cocktail by dounce homogenization. The complex formation was initiated by the addition of $20 \mu \mathrm{M}$ peptide $20,10 \mu \mathrm{g} / \mathrm{mL} \mathrm{Nb35}$ and $25 \mathrm{mU} / \mathrm{mL}$ apyrase. After $1.5 \mathrm{~h}$ incubation at RT, the membrane was solubilized in the buffer above supplemented with $0.5 \%(\mathrm{w} / \mathrm{v}) \mathrm{LMNG}$ and $0.1 \%(\mathrm{w} / \mathrm{v}) \mathrm{CHS}$ for $2 \mathrm{~h}$ at $4{ }^{\circ} \mathrm{C}$. The supernatant was isolated by centrifugation at $65,000 \mathrm{~g}$ for $30 \mathrm{~min}$ and incubated with amylose resin for $2 \mathrm{~h}$ at $4{ }^{\circ} \mathrm{C}$. The resin was then collected by centrifugation at $500 \mathrm{~g}$ for $10 \mathrm{~min}$ and washed in gravity flow column with $5 \mathrm{CVs}$ of buffer containing $20 \mathrm{mM}$ HEPES, pH 7.5, $100 \mathrm{mM} \mathrm{NaCl}, 10 \%$ (v/v) glycerol, $5 \mathrm{mM}$ $\mathrm{MgCl}_{2}, 1 \mathrm{mM} \mathrm{MnCl}, 25 \mu \mathrm{M}$ TCEP, $0.1 \%$ (w/v) LMNG, 0.02\% (w/v) CHS and $5 \mu \mathrm{M}$ peptide 20 , followed by washing with $15 \mathrm{CVs}$ of buffer containing $20 \mathrm{mM}$ HEPES, pH 7.5, $100 \mathrm{mM} \mathrm{NaCl}, 10 \%$ (v/v) glycerol, $5 \mathrm{mM} \mathrm{MgCl}_{2}, 1 \mathrm{mM} \mathrm{MnCl}_{2}$, $25 \mu \mathrm{M}$ TCEP, $0.03 \%(\mathrm{w} / \mathrm{v})$ LMNG, $0.01 \%$ (w/v) GDN, 0.008\% (w/v) CHS and $5 \mu \mathrm{M}$ peptide 20. The protein was then incubated overnight with TEV protease on the column to remove the $\mathrm{C}$-terminal $2 \mathrm{MBP}$-tag in the buffer above at $4^{\circ} \mathrm{C}$. The flow through was collected next day and concentrated with a $100 \mathrm{kDa}$ molecular weight cut-off concentrator. The concentrated product was loaded onto a Superdex 200 increase 10/300 GL column with SEC running buffer containing $20 \mathrm{mM}$ HEPES, pH 7.5, $100 \mathrm{mM} \mathrm{NaCl}, 10 \mathrm{mM} \mathrm{MgCl}_{2}, 100 \mu \mathrm{M}$ TCEP, $2 \mu \mathrm{M}$ peptide $20,0.00075 \%$ LMNG, $0.00025 \%$ GDN and $0.0002 \%(w / v)$ CHS. The fractions for monomeric complex were collected and concentrated to $15-20 \mathrm{mg} / \mathrm{mL}$ for cryo-EM examination.

For the peptide 20-GCGR-G $\mathrm{G}_{\mathrm{s}}$ complex, cell pellets were resuspended in $20 \mathrm{mM}$ HEPES, pH 7.4, $50 \mathrm{mM} \mathrm{NaCl}, 2 \mathrm{mM} \mathrm{MgCl}$ with protease inhibitor cocktail, EDTA-free, $5 \mu \mathrm{M}$ peptide $20,10 \mu \mathrm{g} / \mathrm{mL} \mathrm{Nb35}$ and $25 \mathrm{mU} / \mathrm{mL}$ apyrase. The suspension was incubated at RT for $2 \mathrm{~h}$ to promote the formation of complexes. Membranes were collected by centrifugation $(30,000 \mathrm{rpm})$ at $4{ }^{\circ} \mathrm{C}$ for $30 \mathrm{~min}$, and solubilized in $0.5 \%(\mathrm{w} / \mathrm{v}) \mathrm{LMNG}, 0.1 \%(\mathrm{w} / \mathrm{v})$ CHS, $10 \mu \mathrm{M}$ peptide $20,2 \mathrm{mM} \mathrm{MgCl}_{2}$, $100 \mathrm{U}$ salt active nuclease and $25 \mathrm{mU} / \mathrm{mL}$ apyrase for $2.5 \mathrm{~h}$ at $4{ }^{\circ} \mathrm{C}$. Supernatant was collected by centrifugation at $30,000 \mathrm{rpm}$ for $30 \mathrm{~min}$. The GCGR complex was incubated overnight with anti-HPC4 affinity resin in the presence of $2 \mathrm{mM} \mathrm{CaCl}$ washed with $20 \mathrm{CVs}$ of $20 \mathrm{mM}$ HEPES, $\mathrm{pH} 7.4,100 \mathrm{mM} \mathrm{NaCl}, 2 \mathrm{mM} \mathrm{MgCl}, 2 \mathrm{mM}$ $\mathrm{CaCl}_{2}, 5 \mu \mathrm{M}$ peptide $20,0.02 \%$ (w/v) LMNG and $0.004 \%(\mathrm{w} / \mathrm{v})$ CHS, and eluted with $5 \mathrm{CVs}$ of buffer by adding $6 \mathrm{mM}$ EDTA and $5 \mu \mathrm{M}$ peptide 20 . The complexes were concentrated by a molecular weight cut-off concentrator and separated by SEC on a Superose 6 Increase 10/300GL column with running buffer containing $20 \mathrm{mM}$ HEPES, pH 7.4, $100 \mathrm{mM} \mathrm{NaCl}, 2 \mathrm{mM} \mathrm{MgCl} 2,0.01 \%$ (w/v) LMNG, $0.002 \%$ $(\mathrm{w} / \mathrm{v})$ CHS and $5 \mu \mathrm{M}$ peptide 20 . The complex samples were concentrated to $12-14 \mathrm{mg} / \mathrm{mL}$ for cryo-EM analysis.

Structure determination. To prepare high-quality human $\mathrm{GIPR}-\mathrm{G}_{\mathrm{s}}$ complexes, the receptor's C-terminal forty-five amino acids (Q422-C466) were truncated, and the NanoBiT tethering strategy was applied $25,37,38,53$. To enhance the receptor's expression, a BRIL fusion protein and an optimized maltose binding proteinmaltose binding protein tag (OMBP-MBP $)^{54}$ were added to the $\mathrm{N}$ and $\mathrm{C}$ termini of the receptor to facilitate the receptor stability and expression (Supplementary Fig. 2a). To solve the tirzepatide-GIPR- $\mathrm{G}_{\mathrm{s}}$ complex structure, we introduced one mutation (T345F) to stabilize complex assembly (Supplementary Fig. 3a). This mutation did not affect ligand binding and signaling properties as verified by both cAMP accumulation and receptor binding assays (Supplementary Fig. 2d).

The tirzepatide-GLP-1R-G $\mathrm{G}_{\mathrm{s}}$ complex was prepared using the same NanoBiT technique to achieve good homogeneity and stability as described previously ${ }^{46}$ (Supplementary Fig. 2b). Large-scale purification was performed and the complexes were collected by SEC for cryo-EM studies, with all components of the complex identified in SDS-PAGE of the SEC peak (Supplementary Fig. 3b). Activation of the modified GIPR and GLP-1R constructs by tirzepatide were confirmed by cAMP accumulation and receptor binding assays, showing similar responses to those of the wild-type (WT) receptors (Supplementary Fig. 3e-h). Acylated and non-acylated tirzepatide displayed reduced potencies in eliciting GIPR- or GLP-1R-mediated cAMP responses (Supplementary Fig. 2f, g).

Identical GIPR and GLP-1R constructs were used for the complex structure with peptide 20. Large-scale purification was conducted and the peptide 20-GIPR/ GLP-1R-G $\mathrm{G}_{\mathrm{s}}$ complexes were collected by SEC for cryo-EM studies, with all components of the complex identified in SDS-PAGE of the SEC peak (Supplementary Fig. 4a, b). Activation of the modified GIPR and GLP-1R constructs by peptide 20 were confirmed by cAMP accumulation assays, showing similar responses to those of the WT (Supplementary Fig. 4d, e). To obtain the peptide $20-\mathrm{GCGR}-\mathrm{G}_{\mathrm{s}}$ complexes, 45 residues (H433-F477) were truncated at the C terminus of the receptor followed by a HPC4 $\operatorname{tag}^{28}$ (Supplementary Fig. $2 \mathrm{c}$ ). We used $\mathrm{DNGa}_{\mathrm{s}}{ }^{34,51}$ and $\mathrm{Nb} 35$ that binds across the Ga:G $\beta$ interface ${ }^{55}$ to enhance protein stability. Purified complex was resolved as a monodisperse peak on SEC, with all components of the complex identified in SDS-PAGE of the SEC peak (Supplementary Fig. 4c). The modified GCGR construct had a lower potency than that of the WT but did not significantly affect the binding affinity and cAMP signaling of GCG (Supplementary Fig. 4f).

Data acquisition and image processing. The purified tirzepatide-GIPR $-\mathrm{G}_{\mathrm{s}}-\mathrm{Nb} 35$ complex at a concentration of $18-20 \mathrm{mg} / \mathrm{mL}$ was mixed with $100 \mu \mathrm{M}$ tirzepatide at $4^{\circ} \mathrm{C}$ and applied to glow-discharged holey carbon grids (Quantifoil R1.2/1.3, Au 300 mesh) that were subsequently vitrified by plunging into liquid ethane using a Vitrobot Mark IV (ThermoFisher Scientific). A Titan Krios equipped with a Gatan K3 Summit direct electron detector and serial EM3.7 were used to acquire cryo-EM images. The microscope was operated at $300 \mathrm{kV}$ accelerating voltage, at a nominal magnification of $46,685 \times$ in counting mode, corresponding to a pixel size of $1.071 \AA$. Totally, 5,434 movies were obtained with a defocus range of -1.2 to $-2.2 \mu \mathrm{m}$. An accumulated dose of 80 electrons per $\AA^{2}$ was fractionated into a movie stack of 36 frames.

The purified tirzepatide-GLP-1R- $\mathrm{G}_{\mathrm{s}}-\mathrm{Nb} 35$ complex $(3 \mu \mathrm{L}$ at about $20 \mathrm{mg} / \mathrm{mL})$ was applied to a glow-discharged holey carbon grid (Quantifoil R1.2/1.3) and blotted subsequently. Sample-coated grids were vitrified by plunging into liquid ethane using a Vitrobot Mark IV (ThermoFisher Scientific). Automatic data collection was performed using serial EM3.7 on a Titan Krios equipped with a Gatan K3 Summit direct electron detector. The microscope was operated at $300 \mathrm{kV}$ accelerating voltage, at a nominal magnification of $46,685 \times$ in counting mode, corresponding to a pixel size of $1.071 \AA$. A total of 9,309 movies were obtained with a defocus ranging from -1.2 to $-2.2 \mu \mathrm{m}$. An accumulated dose of 80 electrons per $\AA^{2}$ was fractionated into a movie stack of 45 frames. 
The purified peptide 20-GIPR- $\mathrm{G}_{\mathrm{s}}-\mathrm{Nb} 35$ complex at a concentration of 5-6 mg/ $\mathrm{mL}$ was mixed with $100 \mu \mathrm{M}$ peptide 20 at $4{ }^{\circ} \mathrm{C}$ and applied to glow-discharged holey carbon grids (Quantifoil R1.2/1.3, Au 300 mesh) that were subsequently vitrified by plunging into liquid ethane using a Vitrobot Mark IV (ThermoFisher Scientific). A Titan Krios equipped with a Gatan K3 Summit direct electron detector and serial EM3.7 were used to acquire cryo-EM images. The microscope was operated at $300 \mathrm{kV}$ accelerating voltage, at a nominal magnification of $46,685 \times$ in counting mode, corresponding to a pixel size of 1.071 A . Totally, 3,948 movies were obtained with a defocus range of -1.2 to $-2.2 \mu \mathrm{m}$. An accumulated dose of 80 electrons per $\AA^{2}$ was fractionated into a movie stack of 36 frames.

The purified peptide $20-\mathrm{GLP}-1 \mathrm{R}-\mathrm{G}_{\mathrm{s}}-\mathrm{Nb} 35$ complex $(3.5 \mu \mathrm{L})$ was applied to glow-discharged holey carbon grids (Quantifoil R1.2/1.3, 300 mesh), and subsequently vitrified using a Vitrobot Mark IV (ThermoFisher Scientific) set at $100 \%$ humidity and $4{ }^{\circ} \mathrm{C}$. Cryo-EM images were collected using serial EM3.7 on a Titan Krios microscope (FEI) equipped with Gatan energy filter and K3 direct electron detector. The microscope was operated at $300 \mathrm{kV}$ accelerating voltage and a calibrated magnification of $46,685 \times$ in counting mode, corresponding to a pixel size of $1.071 \AA$. The total exposure time was set to $7.2 \mathrm{~s}$ with intermediate frames recorded every $0.2 \mathrm{~s}$, resulting in an accumulated dose of 80 electrons per $\AA^{2}$ with a defocus range of -1.2 to $-2.2 \mu \mathrm{m}$. Totally, 4,778 images were collected and used for data processing.

The purified peptide $20-\mathrm{GCGR}-\mathrm{G}_{\mathrm{s}}-\mathrm{Nb} 35$ complex at a concentration of $12-14 \mathrm{mg} / \mathrm{mL}$ was mixed with $100 \mu \mathrm{M}$ peptide 20 at $4{ }^{\circ} \mathrm{C}$ and applied to glowdischarged holey carbon grids (Quantifoil R1.2/1.3, Au 300 mesh) that were subsequently vitrified by plunging into liquid ethane using a Vitrobot Mark IV (ThermoFisher Scientific). A Titan Krios equipped with a Gatan K3 Summit direct electron detector and serial EM3.7 were used to acquire cryo-EM images. The microscope was operated at $300 \mathrm{kV}$ accelerating voltage, at a nominal magnification of $46,685 \times$ in counting mode, corresponding to a pixel size of $1.071 \AA$. Totally, 4,620 movies were obtained with a defocus range of -1.2 to $-2.2 \mu \mathrm{m}$. An accumulated dose of 80 electrons per $\AA^{2}$ was fractionated into a movie stack of 36 frames.

The purified non-acylated tirzepatide-GIPR-mini- $\mathrm{G}_{\mathrm{s}}-\mathrm{Nb} 35$ complex at a concentration of $14-16 \mathrm{mg} / \mathrm{mL}$ was mixed with $100 \mu \mathrm{M}$ non-acylated tirzepatide at $4{ }^{\circ} \mathrm{C}$ and applied to glow-discharged holey carbon grids (Quantifoil R1.2/1.3, Au $300 \mathrm{mesh}$ ) that were subsequently vitrified by plunging into liquid ethane using a Vitrobot Mark IV (ThermoFisher Scientific). A Titan Krios equipped with a Gatan K3 Summit direct electron detector and serial EM3.7 were used to acquire cryo-EM images. The microscope was operated at $300 \mathrm{kV}$ accelerating voltage, at a nominal magnification of $46,685 \times$ in counting mode, corresponding to a pixel size of $1.071 \AA$. Totally, 8,159 movies were obtained with a defocus range of -1.2 to $-2.2 \mu \mathrm{m}$. An accumulated dose of 80 electrons per $\AA^{2}$ was fractionated into a movie stack of 36 frames.

The purified non-acylated tirzepatide-GLP-1R-mini- $\mathrm{G}_{\mathrm{s}}-\mathrm{Nb} 35$ complex $(3.5 \mu \mathrm{L}$ ) was applied to glow-discharged holey carbon grids (Quantifoil R1.2/1.3, 300 mesh), and subsequently vitrified using a Vitrobot Mark IV (ThermoFisher Scientific) set at $100 \%$ humidity and $4{ }^{\circ} \mathrm{C}$. Cryo-EM images were collected using serial EM3.7 on a Titan Krios microscope (FEI) equipped with Gatan energy filter and $\mathrm{K} 3$ direct electron detector. The microscope was operated at $300 \mathrm{kV}$ accelerating voltage and a calibrated magnification of $46,685 \times$ in counting mode, corresponding to a pixel size of $1.071 \AA$. The total exposure time was set to $7.2 \mathrm{~s}$ with intermediate frames recorded every $0.2 \mathrm{~s}$, resulting in an accumulated dose of 80 electrons per $\AA^{2}$ with a defocus range of -1.2 to $-2.2 \mu \mathrm{m}$. Totally, 4,778 images were collected and used for data processing.

Dose-fractionated image stacks were subjected to beam-induced motion correction using MotionCor2.156. A sum of all frames, filtered according to the exposure dose, in each image stack was used for further processing. Contrast transfer function parameters for each micrograph were determined by Gctf vl.06 ${ }^{57}$. Automated particle selection and data processing were performed using RELION3.0 beta 258

For the dataset of the tirzepatide-GIPR- $\mathrm{G}_{\mathrm{s}}-\mathrm{Nb} 35$ complex, automated particle selection yielded 4,260,187 particles, which were subjected to reference-free 2D classification, producing 1,771,599 particles with well-defined averages. This subset of particle projections was subjected to a round of 3D classification resulting in one well-defined subset with 870,227 projections. Further 3D classification focusing on the alignment on the whole complex produced one high-quality subset accounting for 511,557 particles. These particles were subsequently subjected to CTF refinement and Bayesian polishing, which generated a map with an indicated global resolution of $3.4 \AA$.

For the dataset of the tirzepatide-GLP-1R-G $-\mathrm{Gb} 35$ complex, automated particle selection yielded 4,213,140 particles, which were subjected to reference-free 2D classification, producing 668,880 particles with well-defined averages. This subset of particle projections was subjected to a round of 3D classification resulting in one well-defined subset with 296,989 projections. Further 3D classification focusing on the alignment on the whole complex produced one high-quality subset accounting for 125,391 particles. These particles were subsequently subjected to CTF refinement and Bayesian polishing, which generated a map with an indicated global resolution of $3.4 \AA$.

For the dataset of the peptide $20-\mathrm{GIPR}-\mathrm{G}_{\mathrm{s}}-\mathrm{Nb} 35$ complex, automated particle selection yielded 5,322,921 particles. The particles were extracted on a binned dataset with a pixel size of $2.142 \AA$ and were subjected to reference-free $2 \mathrm{D}$ classification, producing 4,334,371 particles with well-defined averages. This subset of particle projections was subjected to a round of 3D classification resulting in one well-defined subset with $1,876,783$ projections. Further 3D classifications focusing on the alignment on the whole complex and the receptor produced one highquality subset accounting for 255,256 particles. These particles were subsequently subjected to CTF refinement and Bayesian polishing, which generated a map with an indicated global resolution of $3.1 \AA$.

For the dataset of the peptide $20-\mathrm{GLP}-1 \mathrm{R}-\mathrm{G}_{\mathrm{s}}-\mathrm{Nb} 35$ complex, automated particle selection yielded $4,124,536$ particles, which were subjected to reference-free 2D classification, producing 2,354,838 particles with well-defined averages. This subset of particle projections was subjected to a round of 3D classification resulting in one well-defined subset with $1,523,580$ projections. Further 3D classifications focusing on the alignment on the whole complex and the receptor produced one high-quality subset accounting for 241,786 particles. These particles were subsequently subjected to CTF refinement and Bayesian polishing, which generated a map with an indicated global resolution of $3.0 \AA$

For the dataset of the peptide $20-\mathrm{GCGR}-\mathrm{G}_{\mathrm{s}}-\mathrm{Nb} 35$ complex, automated particle selection yielded 3,931,945 particles, which were subjected to reference-free 2D classification, producing 917,065 particles with well-defined averages. This subset of particle projections was subjected to a round of 3D classification resulting in one well-defined subset with 578,668 projections. Further 3D classification focusing on the alignment on the whole complex produced one high-quality subset accounting for 383,657 particles. These particles were subsequently subjected to CTF refinement and Bayesian polishing, which generated a map with an indicated global resolution of $3.5 \AA$.

For the dataset of the non-acylated tirzepatide-GIPR-mini- $\mathrm{G}_{\mathrm{s}}-\mathrm{Nb} 35$ complex, automated particle selection yielded 7,204,521 particles, which were subjected to reference-free 2D classification, producing 2,718,249 particles with well-defined averages. This subset of particle projections was subjected to a round of 3D classification resulting in one well-defined subset with $2,102,580$ projections. Further 3D classification focusing on the alignment on the whole complex produced one high-quality subset accounting for 1,251,553 particles. These particles were subsequently subjected to CTF refinement and Bayesian polishing, which generated a map with an indicated global resolution of $3.2 \AA$.

For the dataset of the non-acylated tirzepatide-GLP-1R-mini- $\mathrm{G}_{\mathrm{s}}-\mathrm{Nb} 35$ complex, automated particle selection yielded 5,985,110 particles, which were subjected to reference-free $2 \mathrm{D}$ classification, producing $1,723,671$ particles with well-defined averages. This subset of particle projections was subjected to a round of 3D classification resulting in one well-defined subset with 906,824 projections. Further 3D classification focusing on the alignment on the whole complex produced one high-quality subset accounting for 452,921 particles. These particles were subsequently subjected to CTF refinement and Bayesian polishing, which generated a map with an indicated global resolution of $3.0 \AA$

Model building and refinement. The models of the tirzepatide- $\mathrm{GIPR}-\mathrm{G}_{\mathrm{s}}$ complex and peptide $20-\mathrm{GIPR}-\mathrm{G}_{\mathrm{s}}$ complex were built using the cryo-EM structure of the GIP-GIPR-G $\mathrm{G}_{\mathrm{s}}$ complex (PDB code: 7DTY) ${ }^{25}$ as the starting point. The models of the tirzepatide-GLP-1R-G $\mathrm{G}_{\mathrm{s}}$ complex and peptide $20-\mathrm{GLP}-1 \mathrm{R}-\mathrm{G}_{\mathrm{s}}$ complex were built using the cryo-EM structure of the GLP-1-GLP-1R-G $\mathrm{G}_{\mathrm{s}}$ complex (PDB code: $6 \times 18)^{27}$ as the starting point. The model of the peptide $20-G C G R-G_{s}$ complex was built using the cryo-EM structure of the GCG-GCGR-G $\mathrm{G}_{\mathrm{s}}$ complex (PDB code: $6 \mathrm{LMK})^{4}$ as the starting point. The models were docked into the EM density maps using Chimera v1.15 $5^{59}$, followed by iterative manual adjustment and rebuilding in COOT 0.9.6 $6^{60}$. Real space refinement was performed using Phenix $1.16^{61}$. The model statistics were validated with MolProbity v $4.2^{62}$. The final refinement statistics are provided in Supplementary Table 2. Structural figures were prepared in UCSF Chimera v1.15 and PyMOL 2.1 (https://pymol.org/2/).

cAMP accumulation assay. For GIPR, GLP-1R and GCGR, multi-targeting agonist stimulated cAMP accumulation was measured by a LANCE Ultra cAMP kit (PerkinElmer). After $24 \mathrm{~h}$ culture, the transfected cells were seeded into 384well microtiter plates at a density of 3,000 cells per well in HBSS supplemented with $5 \mathrm{mM}$ HEPES, $0.1 \%$ (w/v) BSA or $0.1 \%(\mathrm{w} / \mathrm{v})$ casein and $0.5 \mathrm{mM}$ 3-isobutyl-1methylxanthine. The cells were stimulated with different concentrations of peptide agonists for $40 \mathrm{~min}$ at RT. Eu-cAMP tracer and $\mathrm{ULight}^{\mathrm{TM}}$-anti-cAMP were then diluted by cAMP detection buffer and added to the plates separately to terminate the reaction. Plates were incubated at RT for $1 \mathrm{~h}$ and the fluorescence intensity measured at $620 \mathrm{~nm}$ and $650 \mathrm{~nm}$ by an EnVision multilabel plate reader (PerkinElmer)

Whole-cell binding assay. For GIPR, CHO-K1 cells were cultured in F-12 medium with $10 \%$ FBS and seeded at a density of 30,000 cells/well in Isoplate-96 plates (PerkinElmer). The WT or mutant GIPR was transiently transfected using Lipofectamine 2000 transfection reagent as previous described ${ }^{25}$. They were then washed twice using F-12 with $0.1 \%$ BSA or $0.1 \%$ casein, $33 \mathrm{mM}$ HEPES, and incubated for $2 \mathrm{~h}$ at $37^{\circ} \mathrm{C}$. For homogeneous binding, cells were incubated in binding buffer with a constant concentration of ${ }^{125} \mathrm{I}_{-} \mathrm{GIP}_{1-42}(30 \mathrm{pM}$, PerkinElmer) and increasing concentrations of unlabeled peptide agonists (3.57 pM to $5 \mu \mathrm{M})$ at RT for 3 h. Following incubation, cells were washed three times with ice-cold PBS 
and lysed by addition of $50 \mu \mathrm{L}$ lysis buffer (PBS supplemented with $20 \mathrm{mM}$ Tris$\mathrm{HCl}, 1 \%$ Triton X-100, $\mathrm{pH}$ 7.4). Fifty $\mu \mathrm{L}$ of scintillation cocktail (OptiPhase SuperMix, PerkinElmer) were added and the plates were subsequently counted for radioactivity (counts per minute, CPM) in a MicroBeta ${ }^{2}$ microplate counter (PerkinElmer).

For GLP-1R and GCGR, CHO-K1 cells (30,000 cells/well) were seeded into Isoplate- 96 plates and incubated for $24 \mathrm{~h}$ at $37^{\circ} \mathrm{C}$ in $5 \% \mathrm{CO}_{2}$. The WT or mutant constructs were transiently transfected using Lipofectamine 2000 transfection reagent as previous described ${ }^{25}$. They were then washed twice using F- 12 with $0.1 \%$ BSA or $0.1 \%$ casein, $33 \mathrm{mM}$ HEPES, and incubated for $2 \mathrm{~h}$ at $37^{\circ} \mathrm{C}$. The medium was removed and ${ }^{125}$ I-GLP-1 ${ }_{(7-36)} \mathrm{NH}_{2}(60 \mathrm{pM})$ or ${ }^{125}$ I-GCG $(40 \mathrm{pM})$ (PerkinElmer) and increasing concentrations unlabeled peptide agonists were added for overnight incubation at $4{ }^{\circ} \mathrm{C}$. Cells were washed three times with ice-cold PBS and lysed in PBS with 1\% Triton X-100, $20 \mathrm{mM}$ Tris-HCl. After addition of scintillation cocktail (PerkinElmer), radioactivity (CPM) was counted on a MicroBeta ${ }^{2}$ microplate counter (PerkinElmer). Data were normalized to the WT response and analyzed using three-parameter logistic equation.

Receptor expression. Cell surface expression of GIPR, GLP-1R and GCGR were determined by flow cytometry $24 \mathrm{~h}$ post-transfection in HEK293T cells. Briefly, approximately $2 \times 10^{5}$ cells were blocked with PBS containing $5 \%$ BSA (w/v) at RT for $15 \mathrm{~min}$. After that, cells expressing GIPR and GLP-1R were incubated with 1:300 anti-Flag primary antibody (diluted with PBS containing 5\% BSA, Sigma), and those expressing GCGR were incubated with 1:50 anti-GCGR antibody (diluted with PBS containing 5\% BSA, Abcam) at RT for $1 \mathrm{~h}$. The cells were then washed three times with PBS containing $1 \%$ BSA (w/v) followed by $1 \mathrm{~h}$ incubation with 1:1,000 anti-mouse Alexa Fluor 488 conjugated secondary antibody (diluted with PBS containing 5\% BSA, Invitrogen) at RT in the dark. After washing three times, cells were resuspended in $200 \mu \mathrm{L}$ PBS containing $1 \%$ BSA for detection by NovoCyte (Agilent) utilizing laser excitation and emission wavelengths of $488 \mathrm{~nm}$ and $530 \mathrm{~nm}$, respectively. For each sample, 20,000 cellular events were collected, and the total fluorescence intensity of positive expression cell population was calculated by NovoExpress 1.2.1. Data were normalized to the WT receptor.

$\boldsymbol{\beta}$-arrestin 2 recruitment. HEK293T cells $\left(3.2 \times 10^{6}\right.$ cells $/ 10 \mathrm{~cm}$ plate $)$ were grown for $24 \mathrm{~h}$ before transiently transfection with $15 \mu \mathrm{g}$ plasmid containing GIPR, GLP$1 R$ or GCGR tagged with Rluc8 and $\beta$-arrestin 2 with a Venus-tag at the $N$ terminus at a ratio of 1:9 by Lipofectamine 3000 transfection reagent. After cells were grown for $24 \mathrm{~h}$, transiently transfected cells were then seeded onto poly-Dlysine coated 96-well culture plates (50,000 cells/well) in DMEM with 10\% FBS. Cells were grown for $20 \mathrm{~h}$ before incubation in assay buffer (HBSS supplemented with $10 \mathrm{mM}$ HEPES, $0.1 \%$ BSA or $0.1 \%$ casein, $\mathrm{pH} 7.4$ ) for $30 \mathrm{~min}$ at $37^{\circ} \mathrm{C}$. Coelentrazine-h (Yeasen Biotech) was added to a final concentration of $5 \mu \mathrm{M}$ for $5 \mathrm{~min}$ before bioluminescence resonance energy transfer (BRET) readings were made using an EnVision plate reader (PerkinElmer). BRET baseline measurements were collected for 15 cycles prior to ligand addition. After which, BRET was measured for 55 cycles. The BRET signal (ratio of $535 \mathrm{~nm}$ over $470 \mathrm{~nm}$ emission) was corrected to the baseline and then vehicle-treated condition to determine ligand-induced changes in BRET response. Concentration-response values were obtained from the area-under-the-curve (AUC) of the responses elicited by $\mathrm{GIP}_{1-42}$, GLP-1 $1_{(7-36)} \mathrm{NH}_{2}$, glucagon, tirzepatide, non-acylated tirzepatide, peptide 20 or nonlipidated peptide 20 .

Molecular dynamics simulation. Molecular dynamics (MD) simulation was performed by Gromacs $2020.1^{63}$. The peptide-receptor complexes were prepared by the Protein Preparation Wizard (Schrodinger 2017-4) with G protein and Nb35 removed. The receptors were capped with acetyl and methylamide, and the titratable residues were left in their dominant state at $\mathrm{pH}$ 7.0. The complexes were embedded in a bilayer composed of 195-200 POPC lipids and solvated with $0.15 \mathrm{M}$ $\mathrm{NaCl}$ in explicit TIP3P waters using CHARMM-GUI Membrane Builder v3.2.264. The CHARMM36-CAMP force filed ${ }^{65}$ was adopted for protein, peptides, lipids and salt ions. The 16-carbon acyl chain (palmitoyl; 16:0) covalently attached to the side chain amine of Lys10 in peptide 20 through a $\gamma$-carboxylate spacer and the $\gamma \mathrm{Glu}$ $2 \times \mathrm{OEG}$ linker, and C18 fatty diacid moiety that was acylated on Lys 20 in tirzepatide were modeled with the CHARMM CGenFF small-molecule force field ${ }^{66}$, program version 1.0.0. The Particle Mesh Ewald (PME) method was used to treat all electrostatic interactions beyond a cut-off of $10 \AA$ and the bonds involving hydrogen atoms were constrained using LINCS algorithm ${ }^{67}$. The complex system was first relaxed using the steepest descent energy minimization, followed by slow heating of the system to $310 \mathrm{~K}$ with restraints. The restraints were reduced gradually over 50 ns. Finally, restrain-free production run was carried out for each simulation, with a time step of $2 \mathrm{fs}$ in the NPT ensemble at $310 \mathrm{~K}$ and $1 \mathrm{bar}$ using the Nose-Hoover thermostat and the semi-isotropic Parrinello-Rahman barostat ${ }^{68}$, respectively.

Statistical analysis. All functional data were presented as means \pm standard error of the mean (S.E.M.). Statistical analysis was performed using GraphPad Prism 8 (GraphPad Software). Concentration-response curves were evaluated with a three- parameter logistic equation. The significance was determined with either two-tailed Student's $t$-test or one-way ANOVA. Significant difference is accepted at $P<0.05$.

Reporting summary. Further information on research design is available in the Nature Research Reporting Summary linked to this article.

\section{Data availability}

The raw data underlying Figs. 3d, e, 5g, h, 7b and Supplementary Figs. 2d-g, 3a-l, 4a-f, $9 \mathrm{a}, \mathrm{b}$ are provided as a Source Data file. The atomic coordinates and electron microscopy maps have been deposited in the Protein Data Bank (PDB) under accession codes: 7FIY (tirzepatide-GIPR-G $\mathrm{G}_{\mathrm{s}}$ complex), 7VAB (non-acylated tirzepatide-GIPR-G $\mathrm{G}_{\mathrm{s}}$ complex) 7FIM (tirzepatide-GLP-1R-G complex), 7VBI (non-acylated tirzepatide-GLP-1R-G complex), 7FIN (peptide 20-GIPR-G complex), 7VBH (peptide $20-$ GLP-1R-G complex) and 7V35 (peptide 20-GCGR-G $\mathrm{G}_{\mathrm{s}}$ complex), as well as Electron Microscopy Data Bank (EMDB) under accession codes: EMD-31606 (tirzepatide-GIPR-G s $_{\mathrm{s}}$ complex), EMD-31836 (non-acylated tirzepatide-GIPR-G complex), EMD-31603

(tirzepatide-GLP-1R-G $\mathrm{G}_{\mathrm{s}}$ complex), EMD-31880 (non-acylated tirzepatide-GLP-1R-G complex), EMD-31604 (peptide 20-GIPR-G complex), EMD-31879 (peptide 20-GLP $1 \mathrm{R}-\mathrm{G}_{\mathrm{s}}$ complex) and EMD-31676 (peptide 20-GCGR-G $\mathrm{G}_{\mathrm{s}}$ complex), respectively. All relevant data are available from the authors and/or included in the manuscript or supplemental data. Source data are provided with this paper.

Received: 30 July 2021; Accepted: 1 February 2022;

Published online: 25 February 2022

\section{References}

1. Wootten, D., Miller, L. J., Koole, C., Christopoulos, A. \& Sexton, P. M. Allostery and biased agonism at class B G protein-coupled receptors. Chem. Rev. 117, 111-138 (2017)

2. Hollenstein, K. et al. Insights into the structure of class B GPCRs. Trends Pharm. Sci. 35, 12-22 (2014).

3. Stemmer, K., Finan, B., DiMarchi, R. D., Tschop, M. H. \& Muller, T. D. Insights into incretin-based therapies for treatment of diabetic dyslipidemia. Adv. Drug Deliv. Rev. 159, 34-53 (2020).

4. Qiao, A. et al. Structural basis of Gs and Gi recognition by the human glucagon receptor. Science 367, 1346-1352 (2020).

5. Yuliantie, E. et al. Pharmacological characterization of mono-, dual- and tripeptidic agonists at GIP and GLP-1 receptors. Biochem. Pharm. 177, 114001 (2020).

6. Darbalaei, S. et al. Evaluation of biased agonism mediated by dual agonists of the GLP-1 and glucagon receptors. Biochem. Pharm. 180, 114150 (2020).

7. Christensen, M., Vedtofte, L., Holst, J. J., Vilsboll, T. \& Knop, F. K. Glucosedependent insulinotropic polypeptide: a bifunctional glucose-dependent regulator of glucagon and insulin secretion in humans. Diabetes 60, 3103-3109 (2011).

8. Gault, V. A., Flatt, P. R. \& O’Harte, F. P. M. Glucose-dependent insulinotropic polypeptide analogues and their therapeutic potential for the treatment of obesity-diabetes. Biochem Biophys. Res. Commun. 308, 207-213 (2003).

9. Campbell, J. E. \& Drucker, D. J. Islet alpha cells and glucagon-critical regulators of energy homeostasis. Nat. Rev. Endocrinol. 11, 329-338 (2015).

10. Brandt, S. J., Gotz, A., Tschop, M. H. \& Muller, T. D. Gut hormone polyagonists for the treatment of type 2 diabetes. Peptides 100, 190-201 (2018).

11. Wilding, J. P. H. et al. Once-Weekly Semaglutide in Adults with Overweight or Obesity. N. Engl. J. Med 384, 989 (2021).

12. Frias, J. P. et al. The sustained effects of a dual GIP/GLP-1 receptor agonist, NNC0090-2746, in patients with type 2 diabetes. Cell Metab. 26, 343-352 e342 (2017).

13. Brandt, S. J., Muller, T. D., DiMarchi, R. D., Tschop, M. H. \& Stemmer, K. Peptide-based multi-agonists: a new paradigm in metabolic pharmacology. J. Intern Med 284, 581-602 (2018).

14. Yang, D. et al. G protein-coupled receptors: structure- and function-based drug discovery. Signal Transduct. Target Ther. 6, 7 (2021).

15. Coskun, T. et al. LY3298176, a novel dual GIP and GLP-1 receptor agonist for the treatment of type 2 diabetes mellitus: from discovery to clinical proof of concept. Mol. Metab. 18, 3-14 (2018).

16. Frias, J. P. et al. Tirzepatide versus semaglutide once weekly in patients with type 2 diabetes. N. Engl. J. Med. 385, 503-515 (2021).

17. Frias, J. P. et al. Efficacy and safety of LY3298176, a novel dual GIP and GLP-1 receptor agonist, in patients with type 2 diabetes: a randomised, placebocontrolled and active comparator-controlled phase 2 trial. Lancet 392, 2180-2193 (2018).

18. Alexiadou, K., Anyiam, O. \& Tan, T. Cracking the combination: gut hormones for the treatment of obesity and diabetes. J. Neuroendocrinol. 31, e12664 (2019). 
19. ClinicalTrials.gov. Investigation of Single Ascending Doses of NNC9204-1706 in Male Subjects Being Overweight or With Obesity, https://clinicaltrials.gov/ct2/ show/NCT03095807 (2018).

20. ClinicalTrials.gov. Research Study of a New Medicine (NNC9204-1706) in People With Overweight or Obesity, https://clinicaltrials.gov/ct2/show/ NCT03661879 (2019).

21. Day, J. W. et al. A new glucagon and GLP-1 co-agonist eliminates obesity in rodents. Nat. Chem. Biol. 5, 749-757 (2009).

22. Finan, B. et al. A rationally designed monomeric peptide triagonist corrects obesity and diabetes in rodents. Nat. Med 21, 27-36 (2015).

23. Tschop, M. H. et al. Unimolecular polypharmacy for treatment of diabetes and obesity. Cell Metab. 24, 51-62 (2016).

24. Jall, S. et al. Monomeric GLP-1/GIP/glucagon triagonism corrects obesity, hepatosteatosis, and dyslipidemia in female mice. Mol. Metab. 6, 440-446 (2017).

25. Zhao, F. et al. Structural insights into hormone recognition by the human glucose-dependent insulinotropic polypeptide receptor. Elife 10, e68719 (2021).

26. Wootten, D., Simms, J., Miller, L. J., Christopoulos, A. \& Sexton, P. M. Polar transmembrane interactions drive formation of ligand-specific and signal pathway-biased family B G protein-coupled receptor conformations. Proc. Natl Acad. Sci. USA 110, 5211-5216 (2013).

27. Zhang, X. et al. Differential GLP-1R binding and activation by peptide and non-peptide agonists. Mol. Cell 80, 485-500 e487 (2020).

28. Chang, R. et al. Cryo-electron microscopy structure of the glucagon receptor with a dual-agonist peptide. J. Biol. Chem. 295, 9313-9325 (2020).

29. Lau, J. et al. Discovery of the Once-Weekly Glucagon-Like Peptide-1 (GLP-1) Analogue Semaglutide. J. Med Chem. 58, 7370-7380 (2015).

30. Zhang, X. et al. Structure and dynamics of semaglutide- and taspoglutidebound GLP-1R-Gs complexes. Cell Rep. 36, 109374 (2021).

31. Wu, F. et al. Full-length human GLP-1 receptor structure without orthosteric ligands. Nat. Commun. 11, 1272 (2020).

32. Zhang, H. et al. Structure of the full-length glucagon class B G-proteincoupled receptor. Nature 546, 259-264 (2017).

33. Zhang, Y. et al. Cryo-EM structure of the activated GLP-1 receptor in complex with a G protein. Nature 546, 248-253 (2017).

34. Liang, Y. L. et al. Phase-plate cryo-EM structure of a biased agonist-bound human GLP-1 receptor-Gs complex. Nature 555, 121-125 (2018).

35. Zhao, L. H. et al. Structure and dynamics of the active human parathyroid hormone receptor-1. Science 364, 148-153 (2019).

36. Hilger, D. et al. Structural insights into differences in $\mathrm{G}$ protein activation by family A and family B GPCRs. Science 369, https://doi.org/10.1126/ science.aba3373 (2020).

37. Sun, W. et al. A unique hormonal recognition feature of the human glucagonlike peptide-2 receptor. Cell Res. 30, 1098-1108 (2020).

38. Zhou, F. et al. Structural basis for activation of the growth hormone-releasing hormone receptor. Nat. Commun. 11, 5205 (2020)

39. Willard, F. S. et al. Tirzepatide is an imbalanced and biased dual GIP and GLP-1 receptor agonist. JCI Insight 5, https://doi.org/10.1172/ jci.insight.140532 (2020).

40. Novikoff, A. et al. Spatiotemporal GLP-1 and GIP receptor signaling and trafficking/recycling dynamics induced by selected receptor mono- and dualagonists. Mol. Metab. 49, 101181 (2021).

41. Pabreja, K., Mohd, M. A., Koole, C., Wootten, D. \& Furness, S. G. Molecular mechanisms underlying physiological and receptor pleiotropic effects mediated by GLP-1R activation. Br. J. Pharm. 171, 1114-1128 (2014)

42. Hoare, S. R. Mechanisms of peptide and nonpeptide ligand binding to Class $B$ G-protein-coupled receptors. Drug Disco. Today 10, 417-427 (2005).

43. Zhao, L. H. et al. Differential requirement of the extracellular domain in activation of class B G Protein-coupled Receptors. J. Biol. Chem. 291, 15119-15130 (2016).

44. Siu, F. Y. et al. Structure of the human glucagon class B G-protein-coupled receptor. Nature 499, 444-449 (2013).

45. Song, G. et al. Human GLP-1 receptor transmembrane domain structure in complex with allosteric modulators. Nature 546, 312-315 (2017).

46. Cong, Z. et al. Molecular insights into ago-allosteric modulation of the human glucagon-like peptide-1 receptor. Nat. Commun. 12, 3763 (2021).

47. Samms, R. J., Coghlan, M. P. \& Sloop, K. W. How may GIP enhance the therapeutic efficacy of GLP-1? Trends Endocrinol. Metab. 31, 410-421 (2020).

48. Tuttle, K. R. Breaking new ground with incretin therapy in diabetes. N. Engl. J. Med. 385, 560-561 (2021).

49. Skow, M. A., Bergmann, N. C. \& Knop, F. K. Diabetes and obesity treatment based on dual incretin receptor activation: 'twincretins'. Diabetes Obes. Metab. 18, 847-854 (2016).

50. Dong, M. et al. Structure and dynamics of the active Gs-coupled human secretin receptor. Nat. Commun. 11, 4137 (2020).
51. Liang, Y. L. et al. Dominant negative G proteins enhance formation and purification of agonist-GPCR-G protein complexes for structure determination. ACS Pharm. Transl. Sci. 1, 12-20 (2018).

52. Zhou, F. et al. Molecular basis of ligand recognition and activation of human V2 vasopressin receptor. Cell Res. 31, 929-931 (2021).

53. Duan, J. et al. Cryo-EM structure of an activated VIP1 receptor-G protein complex revealed by a NanoBiT tethering strategy. Nat. Commun. 11, 4121 (2020).

54. Kang, Y. et al. Crystal structure of rhodopsin bound to arrestin by femtosecond X-ray laser. Nature 523, 561-567 (2015).

55. Rasmussen, S. G. et al. Crystal structure of the beta2 adrenergic receptor-Gs protein complex. Nature 477, 549-555 (2011).

56. Zheng, S. Q. et al. MotionCor2: anisotropic correction of beam-induced motion for improved cryo-electron microscopy. Nat. Methods 14, 331-332 (2017).

57. Zhang, K. Gctf: Real-time CTF determination and correction. J. Struct. Biol. 193, 1-12 (2016).

58. Scheres, S. H. RELION: implementation of a Bayesian approach to cryo-EM structure determination. J. Struct. Biol. 180, 519-530 (2012).

59. Pettersen, E. F. et al. UCSF Chimera-a visualization system for exploratory research and analysis. J. Comput Chem. 25, 1605-1612 (2004).

60. Emsley, P. \& Cowtan, K. Coot: model-building tools for molecular graphics. Acta Crystallogr D. Biol. Crystallogr. 60, 2126-2132 (2004).

61. Adams, P. D. et al. PHENIX: a comprehensive Python-based system for macromolecular structure solution. Acta Crystallogr. D. Biol. Crystallogr. 66, 213-221 (2010)

62. Chen, V. B. et al. MolProbity: all-atom structure validation for macromolecular crystallography. Acta Crystallogr. D. Biol. Crystallogr. 66, 12-21 (2010).

63. Abraham, M. J. et al. GROMACS: high performance molecular simulations through multi-level parallelism from laptops to supercomputers. SoftwareX 1-2, 19-25 (2015).

64. Wu, E. L. et al. CHARMM-GUI Membrane Builder toward realistic biological membrane simulations. J. Comput. Chem. 35, 1997-2004 (2014).

65. Guvench, O. et al. CHARMM additive all-atom force field for carbohydrate derivatives and its utility in polysaccharide and carbohydrate-protein modeling. J. Chem. Theory Comput. 7, 3162-3180 (2011).

66. Yu, W., He, X., Vanommeslaeghe, K. \& MacKerell, A. D. Jr Extension of the CHARMM General Force Field to sulfonyl-containing compounds and its utility in biomolecular simulations. J. Comput. Chem. 33, 2451-2468 (2012).

67. Hess, B. P-LINCS: a parallel linear constraint solver for molecular simulation. J. Chem. Theory Comput 4, 116-122 (2008).

68. Aoki, K. M. \& Yonezawa, F. Constant-pressure molecular-dynamics simulations of the crystal-smectic transition in systems of soft parallel spherocylinders. Phys. Rev. A 46, 6541-6549 (1992).

\section{Acknowledgements}

We are grateful to Ms. Jiahui Yan, Drs. Fan Wu and Fulai Zhou for technical assistance and to Drs. Raymond C. Stevens, Radostin Danev, Denise Wootten and Patrick M. Sexton for valuable interactions. This work was partially supported by National Natural Science Foundation of China 81872915 (M.-W.W.), 82073904 (M.-W.W.), 32071203 (L.H.Z), 81922071 (Y.Z.), 81773792 (D.Y.), 81973373 (D.Y.) and 21704064 (Q.T.Z.); National Science and Technology Major Project of China - Key New Drug Creation and Manufacturing Program 2018ZX09735-001 (M.-W.W.) and 2018ZX09711002-002-005 (D.Y.); the National Key Basic Research Program of China 2018YFA0507000 (M.-W.W.) and 2019YFA0508800 (Y.Z.); Ministry of Science and Technology of China 2018YFA0507002 (H.E.X.); Shanghai Municipal Science and Technology Major Project 2019SHZDZX02 (H.E.X.); the Strategic Priority Research Program of Chinese Academy of Sciences XDB37030103 (H.E.X.); Novo Nordisk-CAS Research Fund grant NNCAS2017-1-CC (D.Y.); Zhejiang Province Science Fund for Distinguished Young Scholars LR19H310001 (Y.Z.); Fundamental Research Funds for Central Universities 2019XZZX001-01-06 (Y.Z.); Shanghai Science and Technology Development Funds 18ZR1447800 (L.H.Z.) and 18431907100 (M.-W.W.); The Young Innovator Association of CAS 2018325 (L.H.Z.) and SA-SIBS Scholarship Program (L.H.Z. and D.Y.). The cryoEM data were collected at Cryo-Electron Microscopy Research Center, Shanghai Institute of Materia Medica, Chinese Academy of Sciences.

\section{Author contributions}

F.H.Z., Z.T.C., K.N.H., and C.Z. designed expression constructs, purified the receptor complexes, screened the specimen, prepared the final samples for negative staining, collected cryo-EM data and participated in manuscript preparation. X.Y.Z., A.Y.L. and T.X. conducted map calculation and participated in figure preparation; Q.Q.M., M.W., L.-N.C. and L.H.Z. built the models of the complexes and carried out structural analyses; Q.T.Z. and W.F. conducted MD simulations, comparative structural analysis and figure preparation; A.T.D. and Y.C. performed ligand binding and signaling experiments under the supervision of D.H.Y.; R.L.C. and P.Y.X. participated in method development; Y.Z. and B.W. assisted in structural studies on GLP-1R and GCGR; H.E.X. and M.-W.W. 
initiated the project; Q.T.Z., L.H.Z., H.E.X. and M.-W.W. supervised the studies, analyzed the data and wrote the manuscript with inputs from all co-authors.

\section{Competing interests}

The authors declare no competing interests.

\section{Additional information}

Supplementary information The online version contains supplementary material available at https://doi.org/10.1038/s41467-022-28683-0.

Correspondence and requests for materials should be addressed to Dehua Yang, Lihua Zhao, H. Eric Xu or Ming-Wei Wang.

Peer review information Nature Communications thanks Christopher Reynolds and the other, anonymous, reviewer(s) for their contribution to the peer review of this work.

Reprints and permission information is available at http://www.nature.com/reprints

Publisher's note Springer Nature remains neutral with regard to jurisdictional claims in published maps and institutional affiliations.

(c) (i) Attribution 4.0 International License, which permits use, sharing, adaptation, distribution and reproduction in any medium or format, as long as you give appropriate credit to the original author(s) and the source, provide a link to the Creative Commons license, and indicate if changes were made. The images or other third party material in this article are included in the article's Creative Commons license, unless indicated otherwise in a credit line to the material. If material is not included in the article's Creative Commons license and your intended use is not permitted by statutory regulation or exceeds the permitted use, you will need to obtain permission directly from the copyright holder. To view a copy of this license, visit http://creativecommons.org/ licenses/by/4.0/.

(c) The Author(s) 2022 